\title{
Global regularity and convergence to equilibrium of reaction-diffusion systems with nonlinear diffusion
}

\author{
Klemens Fellner(D, Evangelos Latos and BaO Quoc Tang
}

\begin{abstract}
We study the boundedness and convergence to equilibrium of weak solutions to reaction-diffusion systems with nonlinear diffusion. The nonlinear diffusion is of porous medium type, and the nonlinear reaction terms are assumed to grow polynomially and to dissipate (or conserve) the total mass. By utilising duality estimates, the dissipation of the total mass and the smoothing effect of the porous medium equation, we prove that if the exponents of the nonlinear diffusion terms are high enough, then weak solutions are bounded, locally Hölder continuous and their $L^{\infty}(\Omega)$-norm grows in time at most polynomially. In order to show convergence to equilibrium, we consider a specific class of nonlinear reaction-diffusion models, which describe a single reversible reaction with arbitrarily many chemical substances. By exploiting a generalised logarithmic Sobolev inequality, an indirect diffusion effect and the polynomial in time growth of the $L^{\infty}(\Omega)$-norm, we show an entropy-entropy production inequality which implies exponential convergence to equilibrium in $L^{p}(\Omega)$-norm, for any $1 \leq p<\infty$, with explicit rates and constants.
\end{abstract}

\section{Contents}

1. Introduction and main results

2. Boundedness and local continuity of weak solutions 966

3. Convergence to equilibrium

4. Entropy-entropy production inequality

5. Proof Theorem 1.1: existence of global weak solution to (S)

\section{Introduction and main results}

In this article, we study the boundedness and convergence to equilibrium of weak solutions to reaction-diffusion systems with nonlinear diffusion

$$
\left\{\begin{array}{lll}
\partial_{t} u_{i}-d_{i} \Delta\left(u_{i}^{m_{i}}\right)=f_{i}(u), & x \in \Omega, \quad t>0, & i=1, \ldots, S, \\
d_{i} \nabla\left(u_{i}^{m_{i}}\right) \cdot \vec{n}=0, & x \in \partial \Omega, \quad t>0, & i=1, \ldots, S, \\
u_{i}(x, 0)=u_{i, 0}(x), & x \in \Omega, &
\end{array}\right.
$$

Mathematics Subject Classification: 35B35, 35B40, 35K57, 35Q92

Keywords: Reaction-diffusion systems, Nonlinear diffusion, Porous medium, Convergence to equilibrium, Entropy method. 
with the unknown functions $u=\left(u_{1}, \ldots, u_{S}\right)$ and $u_{i}: \Omega \times \mathbb{R}_{+} \mapsto \mathbb{R}$, the positive diffusion coefficients $d_{i}>0$, the porous medium exponents $m_{i}>1$ and where $\Omega \subset \mathbb{R}^{d}$ denotes a bounded domain with sufficiently smooth boundary $\partial \Omega$ (e.g. $\partial \Omega$ is of class $C^{2+\varrho}$ for some $\varrho>0$ ) with outward unit normal $\vec{n}$ on $\partial \Omega$. Moreover, the conditions imposed on the nonlinear reaction terms $f_{i}(u)$ and the non-negative initial data $u_{i, 0}$ will be specified later.

The first part of this paper considers weak solutions to system (S). Our aim is to provide sufficient conditions on the porous medium exponents $m_{i}$ and on the nonlinearities $f_{i}(u)$, under which weak solutions are indeed bounded in $L^{\infty}$ (and thus locally Hölder continuous) for all times and grow at most polynomially in time. More precisely, we assume the following conditions on the nonlinearities:

(i) The nonlinearities $f_{i}: \mathbb{R}^{S} \rightarrow \mathbb{R}$ are locally Lipschitz functions and satisfy

$$
\left|f_{i}(u)\right| \leq C\left(1+|u|^{\nu}\right), \quad \forall u=\left(u_{1}, \ldots, u_{S}\right) \in \mathbb{R}^{S}, \quad \forall i=1, \ldots, S,
$$

where $\mathbb{R} \ni v \geq 1$ is the maximal growth exponent of the reaction terms.

(ii) There exist positive constants $\lambda_{1}, \ldots, \lambda_{S}>0$ such that:

$$
\sum_{i=1}^{S} \lambda_{i} f_{i}(u) \leq 0, \quad \forall u \in \mathbb{R}^{S},
$$

which formally implies the following mass dissipation law

$$
\frac{\mathrm{d}}{\mathrm{d} t} \int_{\Omega} \sum_{i=1}^{S} \lambda_{i} u_{i} \mathrm{~d} x \leq 0 .
$$

(iii) The nonlinearities are assumed quasi-positive, that is for all $i=1, \ldots, S$, holds

$$
f\left(u_{1}, \ldots, u_{i-1}, 0, u_{i+1}, \ldots, u_{S}\right) \geq 0, \quad \forall u_{1}, \ldots, u_{S} \geq 0
$$

The quasi-positivity condition (P) ensures global non-negativity of solutions subject to non-negative initial data, see e.g. [26,36].

The existence of global weak solutions to (S) subject to homogeneous Dirichlet boundary conditions and under the assumptions $(\mathrm{G})-(\mathrm{M})-(\mathrm{P})$ was recently obtained in [26]. The proof of the following Theorem 1.1 on the existence of weak solutions to $(\mathrm{S})$ subject to Neumann boundary conditions uses similar arguments to [26] and is postponed to Sect. 5 .

Theorem 1.1. Assume the conditions (G), (M) and (P) and consider non-negative initial data $\left(u_{i, 0}\right) \in L^{2}(\Omega)^{S}$. If

$$
m_{i}>\max \{v-1 ; 1\} \text { for all } i=1 \ldots S \text {, }
$$

then, there exists a global weak non-negative solution to system (S) in the sense that, for all $i=1, \ldots, S, u_{i} \in C\left([0,+\infty) ; L^{1}(\Omega)\right), u_{i}^{m_{i}} \in L^{1}\left(0, T ; W^{1,1}(\Omega)\right)$, 


$$
\begin{aligned}
& f_{i}(u) \in L^{1}(\Omega \times[0, T]) \text { and } \\
& \quad-\int_{\Omega} \psi(0) u_{i, 0} d x-\int_{0}^{T} \int_{\Omega}\left(u_{i} \partial_{t} \psi+d_{i} u_{i}^{m_{i}} \Delta \psi\right) d x d t=\int_{0}^{T} \int_{\Omega} \psi f_{i}(u) d x d t
\end{aligned}
$$

for all test function $\psi \in C^{2,1}(\bar{\Omega} \times[0, T])$ with $\nabla \psi \cdot \vec{n}=0$ on $\partial \Omega \times(0, T)$ and $\psi(\cdot, T)=0$.

Moreover, a solution $u=\left(u_{1}, \ldots, u_{S}\right)$ to $(\mathrm{S})$ with $(\mathrm{M})$ and $(\mathrm{P})$ satisfy

$$
\left\|u_{i}\right\|_{L^{m_{i}+1}\left(Q_{T}\right)} \leq C \text { for all } T>0 \text { and } i=1, \ldots, S,
$$

where the constant $C$ depends on the $L^{2}$-norm of the initial data, the constants $\lambda_{i}$ in (M), the diffusion coefficients $d_{i}>0$ and the domain $\Omega$.

Remark 1.1. With a more careful analysis, it seems possible to generalise Theorem 1.1 and consider initial data $u_{i, 0} \in L^{1}(\Omega)$. We refer the interested reader to [38] for the case of systems with quadratic nonlinearities and $L^{1}$ initial data.

Given the weak solutions of Theorem 1.1, our aim is to establish their boundedness and a polynomially in time growing $L^{\infty}$-estimate under stronger assumptions on the porous medium exponents $m_{i}$ : first, we recall the a priori estimate $u_{i} \in L^{m_{i}+1}\left(Q_{T}\right)$ of Theorem 1.1 and the growth condition $(\mathrm{G})$ imply $f_{i}(u) \in L^{1+\varrho}\left(Q_{T}\right)$ for some $\varrho>0$, which also justifies the definition of weak solutions in Theorem 1.1. In fact, the $L^{1+\varrho}$ integrability guarantees uniform integrability of nonlinearities in a suitable approximating scheme (see the proof of Theorem 1.1 in Sect. 5).

Intuitively, Theorem 1.1 states that larger exponents $m_{i}$ yield higher integrability of the nonlinearities $f_{i}(u)$. Moreover, the functions $u_{i}$ solve a porous medium equation with the right-hand side having higher integrability. Thus, by quantifying the smoothing effect from the porous medium equation, this allows to start a bootstrap argument, which eventually leads to boundedness of $u_{i}$ in $L^{\infty}$. In particular, it is of importance that our argument allows to show that the growth in time of the $L^{\infty}$-norms is at most polynomial. The first main result of this article is the following theorem.

Theorem 1.2. (Global bounded weak solutions) Let $\Omega \subset \mathbb{R}^{d}$ be bounded with sufficiently smooth boundary. Let the initial data $0 \leq u_{i, 0} \in L^{\infty}(\Omega)$, assume the conditions $(\mathrm{G}),(\mathrm{M})$ and $(\mathrm{P})$ and $m_{i}>\max \{v-1 ; 1\}$ for all $i=1 \ldots S$ as required by Theorem 1.1. Finally, in dimensions $d \geq 3$, we additionally assume

$$
m_{i}>v-\frac{4}{d+2}, \quad \forall i=1 \ldots S .
$$

Then, any weak solution of (S) obtained in Theorem 1.1 is bounded in $L^{\infty}(\Omega)$ and grows in time at most polynomially in the sense that, for any $T>0$,

$$
\left\|u_{i}\right\|_{L^{\infty}\left(Q_{T}\right)} \leq C_{T}, \quad \forall i=1 \ldots S
$$

where $C_{T}$ is a constant which depends at most polynomially on time. Consequently, these solutions are locally (in $Q_{T}$ ) Hölder continuous, see e.g. [43]. 
Remark 1.2. (Weakened assumptions on mass dissipation and initial data) If one is only interested in the boundedness of solutions but not in the polynomial growth of the $L^{\infty}$-norm, then the mass dissipation condition (M) can in fact be weakened to

$$
\sum_{i=1}^{S} \lambda_{i} f_{i}(u) \leq C_{1} \sum_{i=1}^{S}\left|u_{i}\right|+C_{2} \quad \text { for all } u \in \mathbb{R}^{S},
$$

for some positive constants $C_{1}, C_{2}$.

Also the assumed initial regularity $u_{i, 0} \in L^{\infty}(\Omega)$ is not optimal and could be relaxed to $L^{p}$ integrability for sufficiently large $p$ according to the details of the proof yet at the price of the readability of the Theorem.

Remark 1.3. When $m_{i}=1$, the condition (1) becomes

$$
v<\frac{d+6}{d+2},
$$

which agrees with the results for linear diffusion systems obtained in [8, Proposition 1.4].

Theorem 1.2 contributes to the large literature on global existence and boundedness of solutions to reaction-diffusion systems, which nevertheless poses still many open questions due to the lack of a unified approach (maximum principles do not hold for general systems). The largest part of the available literature, however, considers the case of linear diffusion, i.e. $m_{i}=1$ in system $(\mathrm{S})$. We refer the reader to the extensive review of Pierre [36] and the references therein, in particular [2,4-6, 14, 22$25,31,35,37,39]$

The case of nonlinear diffusion, on the other hand, is much less investigated. Most of the existing results considered special systems with special structures, see e.g. $[28,30,42]$. Up to the best of our knowledge, system (S) under the general structural assumptions (G)-(M)-(P) was only studied very recently in [26], where the authors showed the global existence of weak solutions. Therefore, the present paper serves as the first result to show the boundedness of weak solutions by assuming stronger conditions on porous medium exponents. Moreover, our proof allows to estimate explicitly the growth in time of the $L^{\infty}$-norm, which turns out to be essential in studying the large-time behaviour of solutions in the following second part of the paper.

The second main result of this paper proves exponential convergence to equilibrium for a class of reaction-diffusion systems with porous media diffusion of the form (S), where the nonlinearities model the following reversible reaction with arbitrarily many chemical substances

$$
\alpha_{1} \mathcal{A}_{1}+\cdots+\alpha_{M} \mathcal{A}_{M} \underset{k_{f}}{\stackrel{k_{b}}{\leftrightarrows}} \beta_{1} \mathcal{B}_{1}+\cdots+\beta_{N} \mathcal{B}_{N}
$$

Here, $\alpha_{i}, \beta_{i} \in[1,+\infty)$ are the stoichiometric coefficients of the $M+N$ involved substances $\mathcal{A}_{1}, \ldots, \mathcal{A}_{M}, \mathcal{B}_{1}, \ldots, \mathcal{B}_{N}$ and $k_{f}, k_{b}>0$ are the forward and backward 
reaction rate constants. For simplicity, yet without loss of generality, we assume $k_{f}=$ $k_{b}=1$. By applying mass action kinetics to (2) and by using the short notation

$$
\begin{aligned}
a & =\left(a_{1}, \ldots, a_{M}\right), \quad b=\left(b_{1}, \ldots, b_{N}\right), \quad \alpha=\left(\alpha_{1}, \ldots, \alpha_{M}\right), \quad \beta=\left(\beta_{1}, \ldots, \beta_{N}\right), \\
a^{\alpha} & =\prod_{i=1}^{M} a_{i}^{\alpha_{i}}, \quad b^{\beta}=\prod_{j=1}^{N} b_{j}^{\beta_{j}},
\end{aligned}
$$

we study the following reaction-diffusion system:

$$
\begin{cases}\partial_{t} a_{i}-d_{i} \Delta\left(a_{i}^{m_{i}}\right)=f_{i}(a, b):=-\alpha_{i}\left[a^{\alpha}-b^{\beta}\right], \forall i=1, \ldots, M & x \in \Omega, \quad t>0, \\ \partial_{t} b_{j}-h_{j} \Delta\left(b_{j}^{p_{j}}\right)=g_{j}(a, b):=\beta_{j}\left[a^{\alpha}-b^{\beta}\right], \forall j=1, \ldots, N & x \in \Omega, \quad t>0, \\ d_{i} \nabla\left(a_{i}^{m_{i}}\right) \cdot \vec{n}=0, \quad \forall i=1, \ldots, M, & x \in \partial \Omega, \quad t>0, \\ h_{j} \nabla\left(b_{j}^{p_{j}}\right) \cdot \vec{n}=0, \quad \forall j=1, \ldots, N, & x \in \partial \Omega, \quad t>0, \\ a_{i}(x, 0)=a_{i, 0}(x), \quad \forall i=1, \ldots, M, & x \in \Omega, \\ b_{j}(x, 0)=b_{j, 0}(x), \quad \forall j=1, \ldots, N, & x \in \Omega .\end{cases}
$$

Here, $d_{i}, h_{j}>0$ are diffusion coefficients, and $m_{i}, p_{j}>1$ are nonlinear diffusion exponents. It is clear that $(\mathrm{R})$ is a special case of $(\mathrm{S})$. It is also straightforward to verify condition $(\mathrm{P})$, while condition $(\mathrm{G})$ is satisfied by choosing,

$$
v=\max \left\{\sum_{i=1}^{M} \alpha_{i}, \sum_{j=1}^{N} \beta_{j}\right\} .
$$

Finally condition (M) is a consequence from noting that

$$
\frac{1}{M} \sum_{i=1}^{M} \frac{1}{\alpha_{i}} f_{i}(a, b)+\frac{1}{N} \sum_{j=1}^{N} \frac{1}{\beta_{j}} g_{j}(a, b)=0 .
$$

After having the conditions $(\mathrm{P}),(\mathrm{G})$ and $(\mathrm{M})$ verified, Theorem 1.1 implies the existence of global weak non-negative solutions of system $(\mathrm{R})$ provided

$$
m_{i}, p_{j}>\max \{v-1 ; 1\} \quad \text { for all } i=1 \ldots M, j=1 \ldots N
$$

Moreover by Theorem 1.2, these solutions are bounded in dimensions $d=1,2$, or in dimensions $d \geq 3$ when additionally assuming

$$
m_{i}, p_{j}>v-\frac{4}{d+2} \text { for all } i=1 \ldots M, j=1 \ldots N
$$

By multiplying the equations for $a_{i}$ and $b_{j}$ with $\beta_{j}$ and $\alpha_{i}$, respectively, and by adding the resulting terms, integration by parts with the homogeneous Neumann boundary conditions implies that these solutions satisfy the following mass conservation laws: 


$$
\begin{aligned}
& \beta_{j} \int_{\Omega} a_{i}(x, t) \mathrm{d} x+\alpha_{i} \int_{\Omega} b_{j}(x, t) \mathrm{d} x \\
& =\beta_{j} \int_{\Omega} a_{i, 0}(x) \mathrm{d} x+\alpha_{i} \int_{\Omega} b_{j, 0}(x) \mathrm{d} x=: M_{i j}>0, \quad \forall i, j,
\end{aligned}
$$

amongst which exactly $M+N-1$ linearly independent conservation laws ought to be selected and only the corresponding $M+N-1$ components of the initial mass vector $M_{i j}$ need to be calculated from the initial data.

System $(\mathrm{R})$ possesses for each fixed positive initial mass vector $\left(M_{i j}\right)$ a unique positive detailed balanced equilibrium $\left(a_{\infty}, b_{\infty}\right)=\left(a_{1, \infty}, \ldots, a_{M, \infty}, b_{1, \infty}, \ldots, b_{N, \infty}\right) \in$ $(0, \infty)^{M+N}$, which is the solutions of the following equilibrium equations:

$$
\left\{\begin{array}{l}
\prod_{i=1}^{M} a_{i \infty}^{\alpha_{i}}=\prod_{j=1}^{N} b_{j \infty}^{\beta_{j}}, \\
\beta_{j} a_{i \infty}+\alpha_{i} b_{j \infty}=M_{i j}, \quad \forall i, j
\end{array}\right.
$$

where we recall that the second line constitutes of only $M+N-1$ linearly independent conditions.

To study the convergence to equilibrium for ( $\mathrm{R})$, we will use the so-called entropy method, which recently proved a highly suitable tool in the analysis of the large-time behaviour of dissipative PDE systems. With respect to reaction-diffusion systems with linear diffusion, we refer in particular to [10-13,19,20,33].

The key entropy functional (or in this case the free energy functional) of system (R) is defined by

$$
E[a, b]=\sum_{i=1}^{M} \int_{\Omega}\left(a_{i} \ln a_{i}-a_{i}+1\right) \mathrm{d} x+\sum_{j=1}^{N} \int_{\Omega}\left(b_{j} \ln b_{j}-b_{j}+1\right) \mathrm{d} x
$$

which dissipates according to the non-negative entropy production functional, that is formally

$$
\begin{aligned}
-\frac{\mathrm{d}}{\mathrm{d} t} E[a, b]=: D[a, b]= & \sum_{i=1}^{M} d_{i} \int_{\Omega} \frac{\left|\nabla a_{i}\right|^{2}}{a_{i}^{2-m_{i}}} \mathrm{~d} x+\sum_{j=1}^{N} h_{j} \int_{\Omega} \frac{\left|\nabla b_{j}\right|^{2}}{b_{j}^{2-p_{j}}} \mathrm{~d} x \\
& +\int_{\Omega}\left(a^{\alpha}-b^{\beta}\right) \ln \frac{a^{\alpha}}{b^{\beta}} \mathrm{d} x \geq 0 .
\end{aligned}
$$

In the case of linear diffusion, i.e. $m_{i}=p_{j}=1$ for all $i=1 \ldots M, j=1 \ldots N$, the convergence to equilibrium of solutions of (R) (or some special cases) was recently studied in e.g. $[10,12,19,33,40]$.

Let us briefly review the entropy method used in the case of linear diffusion and then highlight the difficulties to be overcome in the current paper when dealing with nonlinear diffusion. In the case of linear diffusion, the entropy production writes as 


$$
\begin{aligned}
D_{\text {lin }}[a, b]= & \sum_{i=1}^{M} d_{i} \int_{\Omega} \frac{\left|\nabla a_{i}\right|^{2}}{a_{i}} \mathrm{~d} x+\sum_{j=1}^{N} h_{j} \int_{\Omega} \frac{\left|\nabla b_{j}\right|^{2}}{b_{j}} \mathrm{~d} x \\
& +\int_{\Omega}\left(a^{\alpha}-b^{\beta}\right) \ln \frac{a^{\alpha}}{b^{\beta}} \mathrm{d} x \geq 0
\end{aligned}
$$

and the entropy method consists in establishing a functional inequality of the form

$$
D_{l i n}[a, b] \geq \lambda\left(E[a, b]-E\left[a_{\infty}, b_{\infty}\right]\right)
$$

for all functions $a=\left(a_{i}\right), b=\left(b_{j}\right)$ satisfying the conservation laws (3). In order to do that, one first uses an additivity property of the relative entropy to calculate

$$
\begin{aligned}
E[a, b]-E\left[a_{\infty}, b_{\infty}\right]= & {\left[\sum_{i=1}^{M} \int_{\Omega} a_{i} \log \frac{a_{i}}{\bar{a}_{i}} \mathrm{~d} x+\sum_{j=1}^{N} \int_{\Omega} b_{j} \log \frac{b_{j}}{\bar{b}_{j}} \mathrm{~d} x\right] } \\
+ & {\left[\sum_{i=1}^{M}\left(\bar{a}_{i} \log \frac{\bar{a}_{i}}{a_{i, \infty}}-\bar{a}_{i}+a_{i, \infty}\right)\right.} \\
& \left.+\sum_{j=1}^{N}\left(\bar{b}_{j} \log \frac{\bar{b}_{j}}{b_{j, \infty}}-\bar{b}_{j}+b_{j, \infty}\right)\right] \\
= & : I_{1}+I_{2} .
\end{aligned}
$$

The term $I_{1}$ is controlled in terms of the entropy production $D_{\text {lin }}[a, b]$ thanks to the logarithmic Sobolev inequality (LSI)

$$
\int_{\Omega} \frac{|\nabla f|^{2}}{f} \mathrm{~d} x \geq C_{\mathrm{LSI}} \int_{\Omega} f \log \frac{f}{\bar{f}} \mathrm{~d} x \quad \text { for all } \quad 0 \leq f \in H^{1}(\Omega) .
$$

The remain term $I_{2}$ only involves the averages of the concentrations $\bar{a}_{i}, \bar{b}_{j}$ and can be controlled by $D_{\text {lin }}[a, b]$ through lengthly, technical, but constructive estimates (see e.g. $[19,40]$ for more details). Note that this entropy approach applies successfully to more complex chemical reaction networks than $(\mathrm{R})$, see [13,20,32,33]. We emphasise that the logarithmic Sobolev inequality (5) is not only used to control the term $I_{1}$ but also plays an important role in the estimates controlling the term $I_{2}$.

In the case of nonlinear diffusion as here considered, we need a generalisation of the LSI (5) to exponents $m_{i}, p_{j} \geq 1$. In this paper, we utilise the following generalisation (see e.g. [34]): for any $m>(d-2)_{+} / d$ with $(d-2)_{+}=\max \{d-2 ; 0\}$, there exists a constant $C(\Omega, m)>0$ such that

$$
\int_{\Omega} \frac{|\nabla f|^{2}}{f^{2-m}} \mathrm{~d} x \geq C(\Omega, m) \bar{f}^{m-1} \int_{\Omega} f \log \frac{f}{\bar{f}} \mathrm{~d} x .
$$

When $m=1$, this coincides with the classical logarithmic Sobolev inequality (5). For system (R), we have in particular 


$$
\begin{aligned}
& \int_{\Omega} \frac{\left|\nabla a_{i}\right|^{2}}{a_{i}^{2-m_{i}}} \mathrm{~d} x \geq C\left(\Omega, m_{i}\right) \bar{a}_{i}^{m_{i}-1} \int_{\Omega} a_{i} \log \frac{a_{i}}{\bar{a}_{i}} \mathrm{~d} x \quad \text { and } \\
& \int_{\Omega} \frac{\left|\nabla b_{j}\right|^{2}}{b_{j}^{2-p_{j}}} \mathrm{~d} x \geq C\left(\Omega, p_{j}\right) \bar{b}_{j}^{p_{j}-1} \int_{\Omega} b_{j} \log \frac{b_{j}}{\bar{b}_{j}} \mathrm{~d} x .
\end{aligned}
$$

Note that if we assume the averages $\bar{a}_{i}$ and $\bar{b}_{j}$ to be bounded below by a positive constant, then one can apply the same strategy as for the linear diffusion case in order to obtain the convergence to equilibrium. However, there is no chemical/physical reason for such a lower bound to hold in the transient behaviour of system (R) subject to general initial data. There are even perfectly admissible initial conditions, where some averages are zero since the corresponding species have not yet been formed.

To overcome this difficulty, we first observe that the mass conservation laws (3) subject to a positive mass vector $M_{i, j}>0$ imply that the averages $\bar{a}_{i}$ and $\bar{b}_{j}$ cannot be simultaneously small. Thus, at any fixed time, at least one of the inequalities in (6) is useful, since either $\bar{a}_{i} \geq \varepsilon$ or $\bar{b}_{j} \geq \varepsilon$ for some suitably chosen $\varepsilon>0$ depending on $M_{i, j}>0$. Secondly, we are able to compensate the still lacking lower bounds in (6) by a phenomena which can be called "indirect diffusion effect" and which means in our context that the reversible reaction (2) transfers diffusion from a species $a_{i}$ (with strictly positive diffusion bound in (6) due to $\bar{a}_{i} \geq \varepsilon$ ) to other species $b_{j}$ (with lacking positive lower diffusion bound) in terms of a functional inequality, see Lemma 3.2 below.

Examples of indirect diffusion effect inequalities were already derived in e.g. [11, $17,18]$, yet typically with a proof which requires uniform in time $L^{\infty}$-bounds on the solutions, which is a severe technical restriction as $L^{\infty}$-bounds for general reactiondiffusion systems are often unknown due to the lack of comparison principles. Note that also the $L^{\infty}$-bounds of Theorem 1.1 would be insufficient since polynomially growing and not uniform in time.

In this work, we are able to prove an indirect diffusion functional inequality without using any $L^{\infty}$-bounds on solutions but instead by exploiting the special structure of (R), see Lemma 3.2. Nevertheless, in the remaining part of applying the entropy method, the polynomial growth in time of the $L^{\infty}$-norm of Theorem 1.2 is still needed in one estimate concerning the relative entropy, yet the $L^{\infty}$-norm appears only within a logarithm. While it is unclear to us whether this is essential or just technical necessary in our approach, it allows to derive a time-dependent entropy-entropy production inequality (as a generalisation of the functional inequality (4)) of the form

$$
D[a(T), b(T)] \geq \Theta(T)\left(E[a(T), b(T)]-E\left[a_{\infty}, b_{\infty}\right]\right) \text { for all } \quad T>0,
$$

where the function $\Theta: \mathbb{R}_{+} \rightarrow \mathbb{R}_{+}$is of order $1 / \ln (1+T)$ and satisfies $\int_{0}^{+\infty} \Theta(\tau) d \tau=$ $+\infty$. Thus, a classical Gronwall argument implies explicit algebraic decay of $E[a(T), b(T)]-E\left[a_{\infty}, b_{\infty}\right]$ to zero and thus algebraic convergence to equilibrium in relative entropy. 
To obtain exponential from algebraic decay, we show that after some sufficiently large time $T_{0}>0$, the averages $\bar{a}_{i}(T)$ and $\bar{b}_{j}(T)$ are bounded below by a positive constant for all $T \geq T_{0}$ (since the equilibrium $\left(a_{\infty}, b_{\infty}\right)$ consists of positive constants). Hence, for $T \geq T_{0}$, we can use the inequalities (6) like in the case for systems with linear diffusion and obtain accordingly exponential convergence to equilibrium. Finally, since $T_{0}$ can be explicitly estimated, one recovers global exponential convergence to equilibrium (i.e. for all $T \geq 0$ ) at the price of a smaller, yet explicit constant. Hence, the second main result of this paper is the following theorem.

Theorem 1.3. Let $\Omega \subset \mathbb{R}^{d}$ be bounded with sufficiently smooth boundary. Consider system $(\mathrm{R})$ - which satisfies the conditions $(\mathrm{G}),(\mathrm{M})$ and $(\mathrm{P})$ - subject to non-negative initial data $a_{i, 0}, b_{j, 0} \in L^{\infty}(\Omega)$. Assume for all $i=1 \ldots M, j=1 \ldots N$ that

$$
m_{i}, p_{j}>\max \{v-1 ; 1\}, \quad \text { where } v=\max \left\{\sum_{i=1}^{M} \alpha_{i}, \sum_{j=1}^{N} \beta_{j}\right\}
$$

Moreover, in dimensions $d \geq 3$, we additionally assume

$$
m_{i}, p_{j}>v-\frac{4}{d+2}, \quad \text { for all } i=1 \ldots M, j=1 \ldots N .
$$

Finally, consider a positive initial mass vector $M_{i j}>0$, which uniquely determines $a$ positive equilibrium $\left(a_{i \infty}, b_{j \infty}\right)$ of system $(\mathrm{R})$.

Then, the bounded global weak solutions of Theorem 1.2 converge exponentially to $\left(a_{\infty}, b_{\infty}\right)$ in all $L^{p}$-norms for $1 \leq p<\infty$, that is

$$
\sum_{i=1}^{M}\left\|a_{i}(t)-a_{i \infty}\right\|_{L^{p}(\Omega)}+\sum_{j=1}^{N}\left\|b_{j}(t)-b_{j \infty}\right\|_{L^{p}(\Omega)} \leq C e^{-\lambda_{p} t}
$$

where the constant $C>0$ and the convergence rate $\lambda_{p}>0$ can be computed explicitly.

Remark 1.4. We remark that in Theorem 1.3, we showed the convergence to equilibrium in any $L^{p}$-norm with $p<\infty$. In the case of linear diffusion, i.e. $m_{i}=1$ for all $i=1, \ldots, S$, we are able to get the exponential convergence to equilibrium in $L^{\infty}$. norm thanks to the Duhamel formula for semilinear equations, see [16, Proof of Theorem 5.1] (see also [21] for local stability in $L^{\infty}$-norm). This technique is not applicable for nonlinear diffusion, and therefore, the question of global stability in $L^{\infty}$-norm for (S) remains as an interesting open problem.

\section{Notation:}

- We denote by $\|\cdot\|$ the usual norm of $L^{2}(\Omega)$. For other $1 \leq p<+\infty$, we write $\|\cdot\|_{p}$ as the norm of $L^{p}(\Omega)$.

- For any $T>0, Q_{T}=\Omega \times(0, T)$ and $L^{p}\left(Q_{T}\right)=: L^{p}\left(0, T ; L^{p}(\Omega)\right)$. The space-time norm is defined as usual

$$
\|f\|_{L^{p}\left(Q_{T}\right)}^{p}=\int_{0}^{T} \int_{\Omega}|f(x, t)|^{p} \mathrm{~d} x \mathrm{~d} t .
$$


- Throughout this work, we will denote by $C_{T}$ a generic positive constant which depends on certain parameters, and more importantly $C_{T}$ grows at most polynomially, i.e. there exists a polynomial $P(x)$ such that $C_{T} \leq P(T)$ for all $T>0$.

Organisation of the paper: Sect. 2 states the proof of Theorem 1.2. The proof of Theorem 1.3 is detailed in Sect. 3. This proof uses also a previously proven entropy-entropy production estimate for reaction-diffusion systems with linear diffusion, which is recalled in Sect. 4 for the sake of completeness. Finally, the existence of global weak solution is stated in Sect. 5.

\section{Boundedness and local continuity of weak solutions}

In this section, we prove for sufficiently large diffusion exponents $m_{i}$ that the weak solutions obtained in Theorem 1.1 are actually bounded in $L^{\infty}$ and thus locally Hölder continuous. In Lemma 2.1, we devise a bootstrap argument for the inhomogeneous porous media equation which proves that if the porous media exponents $m_{i}$ and the initial integrability are high enough, then the weak solutions of Theorem 1.1 satisfy an improve integrability in a space $L^{s}\left(Q_{T}\right)$ and the $L^{s}$-norm grows at most polynomially in time $T$.

Lemma 2.1. (Smoothing effect of porous medium equation) Suppose that $m \geq 1$. Assume $f \in L^{p_{0}}\left(Q_{T}\right)$ for some $p_{0}>1$ with $\|f\|_{L^{p_{0}}\left(Q_{T}\right)} \leq C_{T}$. Let $u$ be the weak solution to the inhomogeneous porous medium equation with positive diffusion coefficient $\delta>0$

$$
\left\{\begin{array}{lll}
\partial_{t} u-\delta \Delta\left(|u|^{m-1} u\right)=f, & x \in \Omega, & t>0, \\
\delta \nabla\left(|u|^{m-1} u\right) \cdot \vec{n}=0, & x \in \partial \Omega, & t>0, \\
u(x, 0)=u_{0}(x), & x \in \Omega, &
\end{array}\right.
$$

and subject to initial data $u_{0} \in L^{\infty}(\Omega)$. Then, $u$ satisfies

$$
\|u\|_{L^{r}\left(Q_{T}\right)} \leq C_{T}, \quad \forall r \in[1, s)
$$

where

$$
s= \begin{cases}+\infty, & \text { if } \quad p_{0} \geq \frac{d+2}{2}, \\ \frac{(m d+2) p_{0}}{d+2-2 p_{0}}, & \text { if } \quad p_{0}<\frac{d+2}{2},\end{cases}
$$

and with a constant $C_{T}$, which only depends on $q, d, m, \Omega$ and at most polynomially on $T$.

Remark 2.1. In the linear case $m=1$, Lemma 2.1 recovers the corresponding regularity estimates of the heat equation, see [8]. While the smoothing effect stated in Lemma 2.1 is certainly well known, our main contribution here lies in the polynomial growth in time of the norms, which will be crucial in Sect. 3. 
Proof. The existence of the weak solution to (8) can be obtained by standard techniques [43, Chapter 11] so we omit it here. The idea of the proof of this lemma follows [8, Lemma 3.3] and is divided into several steps.

Step 1. Let $\mu>1$. By multiplying (8) by $\mu|u|^{\mu-1} \operatorname{sign}(u)$ (more precisely by multiplying with a smoothed version of the modulus $|u|$ and its derivative $\operatorname{sign}(u)$ and letting then the smoothing tend to zero) then integrating over $\Omega$, we obtain

$$
\frac{\mathrm{d}}{\mathrm{d} t}\|u\|_{\mu}^{\mu}-\delta \mu \int_{\Omega} \Delta\left(|u|^{m-1} u\right)|u|^{\mu-1} \operatorname{sign}(u) \mathrm{d} x=\mu \int_{\Omega} f|u|^{\mu-1} \operatorname{sign}(u) \mathrm{d} x .
$$

Integration by parts and the homogeneous Neumann boundary condition $\nabla\left(|u|^{m-1} u\right)$. $\vec{n}=0$ lead to

$$
\begin{aligned}
& -\delta \mu \int_{\Omega} \Delta\left(|u|^{m-1} u\right)|u|^{\mu-1} \operatorname{sign}(u) \mathrm{d} x \\
& \geq m(\mu-1) \mu \delta \int_{\Omega}|u|^{m+\mu-3}|\nabla u|^{2} \mathrm{~d} x+m \mu \delta \int_{\Omega}|u|^{m+\mu-2}|\nabla u|^{2} \mathrm{~d} x \\
& \geq \underbrace{\frac{4 m(\mu-1) \mu \delta}{(m+\mu-1)^{2}}}_{=: C(\mu)} \int_{\Omega}\left|\nabla\left(|u|^{\frac{m+\mu-1}{2}}\right)\right|^{2} \mathrm{~d} x .
\end{aligned}
$$

By Hölder's inequality

$$
\left.\left|\mu \int_{\Omega} f\right| u\right|^{\mu-1} \operatorname{sign}(u) \mathrm{d} x \mid \leq \mu\|f\|_{p_{0}}\|u\|_{\frac{p_{0}(\mu-1)}{p_{0}-1}}^{\mu-1} .
$$

Therefore, it follows from (9) that

$$
\frac{\mathrm{d}}{\mathrm{d} t}\|u\|_{\mu}^{\mu}+C(\mu) \int_{\Omega}\left|\nabla\left(|u|^{\frac{m+\mu-1}{2}}\right)\right|^{2} \mathrm{~d} x \leq \mu\|f\|_{p_{0}}\|u\|_{\frac{p_{0}(\mu-1)}{p_{0}-1}}^{\mu-1} .
$$

Step 2. Choose $\mu=p_{0}>1$ in (10), we get

$$
\frac{\mathrm{d}}{\mathrm{d} t}\|u\|_{p_{0}}^{p_{0}}+C\left(p_{0}\right) \int_{\Omega}\left|\nabla\left(|u|^{\frac{m+p_{0}-1}{2}}\right)\right|^{2} \mathrm{~d} x \leq p_{0}\|f\|_{p_{0}}\|u\|_{p_{0}}^{p_{0}-1} .
$$

By applying for $r<1$ the elementary inequality

$$
y^{\prime} \leq \alpha(t) y^{1-r} \Longrightarrow y(T) \leq\left[y(0)^{r}+r \int_{0}^{T} \alpha(t) \mathrm{d} t\right]^{1 / r},
$$

to (11) with $r=1 / p_{0}$ and $y(t)=\|u(t)\|_{p_{0}}^{p_{0}}$, we obtain

$$
\begin{aligned}
\|u(T)\|_{p_{0}}^{p_{0}} & \leq\left[\left\|u_{0}\right\|_{p_{0}}+\int_{0}^{T}\|f\|_{p_{0}} \mathrm{~d} t\right]^{p_{0}} \\
& \leq\left[\left\|u_{0}\right\|_{p_{0}}+\|f\|_{L^{p_{0}\left(Q_{T}\right)}} T^{\left(p_{0}-1\right) / p_{0}}\right]^{p_{0}}=: C_{T, 0} .
\end{aligned}
$$


That means

$$
u \in L^{\infty}\left(0, T ; L^{p_{0}}(\Omega)\right) \quad \text { and } \quad\|u(T)\|_{p_{0}}^{p_{0}} \leq C_{T, 0}
$$

with $C_{T, 0}$ is defined in (13) grows at most polynomially in $T$. By integrating (11) with respect to $t$ on $(0, T)$ and by using Young's inequality and the convention $r_{0}:=m+$ $p_{0}-1>1$, we get

$$
\begin{aligned}
C\left(p_{0}\right) \int_{0}^{T} \int_{\Omega}\left|\nabla\left(|u|^{\frac{r_{0}}{2}}\right)\right|^{2} \mathrm{~d} x \mathrm{~d} t & \leq\left\|u_{0}\right\|_{p_{0}}^{p_{0}}+p_{0} \int_{0}^{T}\|f\|_{p_{0}}\|u\|_{p_{0}}^{p_{0}-1} \mathrm{~d} t \\
& \leq\left\|u_{0}\right\|_{p_{0}}^{p_{0}}+p_{0}\|f\|_{L^{p_{0}\left(Q_{T}\right)}\|u\|_{L^{p_{0}}\left(Q_{T}\right)}^{p_{0}-1}} .
\end{aligned}
$$

By adding $\left.\left.C\left(p_{0}\right) \int_{0}^{T} \int_{\Omega}|| u\right|^{\frac{r_{0}}{2}}\right|^{2} \mathrm{~d} x \mathrm{~d} t$ to both sides, we have

$$
\begin{aligned}
C\left(p_{0}\right) \int_{0}^{T}\left\||u|^{\frac{r_{0}}{2}}\right\|_{H^{1}(\Omega)}^{2} \mathrm{~d} t= & C\left(p_{0}\right) \int_{0}^{T}\left[\int_{\Omega}\left|\nabla\left(|u|^{\frac{r_{0}}{2}}\right)\right|^{2} \mathrm{~d} x+\left.\left.\int_{\Omega}|| u\right|^{\frac{r_{0}}{2}}\right|^{2} \mathrm{~d} x\right] \mathrm{d} t \\
\leq & \left\|u_{0}\right\|_{p_{0}}^{p_{0}}+p_{0}\|f\|_{L^{p_{0}}\left(Q_{T}\right)}\|u\|_{L^{p_{0}}\left(Q_{T}\right)}^{p_{0}-1} \\
& +C\left(p_{0}\right) \int_{0}^{T}\|u\|_{r_{0}}^{r_{0}} \mathrm{~d} t .
\end{aligned}
$$

By the Sobolev's embedding, we have

$$
\begin{aligned}
C\left(p_{0}\right) \int_{0}^{T}\left\||u|^{\frac{r_{0}}{2}}\right\|_{H^{1}(\Omega)}^{2} & \geq C\left(p_{0}\right) C_{S}^{2} \int_{0}^{T}\|u\|_{s_{0}}^{r_{0}} \mathrm{~d} t \quad \text { with } \\
s_{0} & = \begin{cases}\frac{r_{0} d}{d-2} & \text { if } d \geq 3, \\
r_{0}<s_{0}<\infty \text { arbitrary } & \text { if } d=1,2 .\end{cases}
\end{aligned}
$$

On the other hand, by using the bound $\|u(t)\|_{p_{0}}^{p_{0}} \leq C_{T, 0}$ in (14) and the interpolation inequality

$$
\begin{aligned}
\|u\|_{r_{0}} & \leq\|u\|_{p_{0}}^{\gamma}\|u\|_{s_{0}}^{1-\gamma} \leq C_{T, 0}^{\gamma / p_{0}}\|u\|_{s_{0}}^{1-\gamma} \text { with } \frac{1}{r_{0}}=\frac{\gamma}{p_{0}}+\frac{1-\gamma}{s_{0}} \text { for } \\
\gamma & =\frac{2 p_{0}}{2 p_{0}+(m-1) d} \in(0,1],
\end{aligned}
$$

we estimate in the cases $m>1$ for which $\gamma<1$

$$
\begin{aligned}
C\left(p_{0}\right) \int_{0}^{T}\|u\|_{r_{0}}^{r_{0}} \mathrm{~d} t & \leq C\left(p_{0}\right) \int_{0}^{T} C_{T, 0}^{\gamma r_{0} / p_{0}}\|u\|_{s_{0}}^{(1-\gamma) r_{0}} \mathrm{~d} t \\
& \leq \frac{C\left(p_{0}\right) C_{S}^{2}}{2} \int_{0}^{T}\|u\|_{s_{0}}^{r_{0}} \mathrm{~d} t+C C_{T, 0}^{r_{0} / p_{0}} T,
\end{aligned}
$$

where we have used Young's inequality (with the exponents $1=(1-\gamma)+\gamma$ ) in the last step. Note that if $m=1$, the bound (17) holds still true yet without the first term and with $r_{0} / p_{0}=1$. Inserting (16) and (17) into (15) leads to 


$$
\begin{aligned}
\int_{0}^{T}\|u\|_{S_{0}}^{r_{0}} \mathrm{~d} t & \leq \frac{2}{C\left(p_{0}\right) C_{S}^{2}}\left[\left\|u_{0}\right\|_{p_{0}}^{p_{0}}+p_{0}\|f\|_{L^{p_{0}}\left(Q_{T}\right)}\|u\|_{L^{p_{0}}\left(Q_{T}\right)}^{p_{0}-1}+C C_{T, 0}^{r_{0} / p_{0}} T\right] \\
& \leq \frac{2}{C\left(p_{0}\right) C_{S}^{2}}\left[\left\|u_{0}\right\|_{p_{0}}^{p_{0}}+p_{0}\|f\|_{L^{p_{0}}\left(Q_{T}\right)}\left(T C_{T, 0}\right)^{\frac{p_{0}-1}{p_{0}}}+C C_{T, 0}^{r_{0} / p_{0}} T\right] \\
& =: D_{T, 0} \quad \text { (use (14)). }
\end{aligned}
$$

It follows that

$$
u \in L^{r_{0}}\left(0, T ; L^{s_{0}}(\Omega)\right) \quad \text { with } \begin{cases}s_{0}=\frac{r_{0} d}{d-2} & \text { if } d \geq 3, \\ r_{0}<s_{0}<\infty \text { arbitrary } & \text { if } d=1,2,\end{cases}
$$

and

$$
\int_{0}^{T}\|u\|_{S_{0}}^{r_{0}} \mathrm{~d} t \leq D_{T, 0}
$$

with $D_{T, 0}$ defined in (18).

Next, we construct a sequence $p_{n} \geq 1$ based on the estimate (14) and (19) such that

$$
\|u(T)\|_{p_{n}}^{p_{n}} \leq C_{T, n}
$$

and

$$
\begin{aligned}
& \int_{0}^{T}\|u\|_{s_{n}}^{r_{n}} \mathrm{~d} t \leq D_{T, n} \quad \text { with } \quad r_{n}=m+p_{n}-1 \quad \text { and } \\
& \begin{cases}s_{n}=\frac{r_{n} d}{d-2} & \text { if } d \geq 3, \\
r_{n}<s_{n}<\infty \text { arbitrary } & \text { if } d=1,2,\end{cases}
\end{aligned}
$$

in which $C_{T, n}$ and $D_{T, n}$ are constants growing at most polynomially in $T$.

Step 3 (Iteration of (20)). In (10), we set $\mu=p_{n+1}$ for $p_{n+1}$ to be chosen later. Thus, we have

$$
\frac{\mathrm{d}}{\mathrm{d} t}\|u\|_{p_{n+1}}^{p_{n+1}}+C\left(p_{n+1}\right) \int_{\Omega}\left|\nabla\left(|u|^{\frac{r_{n+1}}{2}}\right)\right|^{2} \mathrm{~d} x \leq p_{n+1}\|f\|_{p_{0}}\|u\|_{\frac{p_{0}\left(p_{n+1}-1\right)}{p_{0}-1}}^{p_{n+1}-1},
$$

where we recall that $r_{n+1}=m+p_{n+1}-1$. By $L^{p}$ - interpolation, we have

$$
\|u\|_{\frac{p_{0}\left(p_{n+1}-1\right)}{p_{0}-1}} \leq\|u\|_{p_{n+1}}^{1-\theta}\|u\|_{s_{n}}^{\theta}
$$

and where $p_{n+1}>1$ has to be chosen such that $\frac{p_{0}\left(p_{n+1}-1\right)}{p_{0}-1} \in\left(p_{n+1}, s_{n}\right)$ with $p_{n+1}<$ $s_{n}$, which entails $\theta \in(0,1)$ in

$$
\frac{p_{0}-1}{p_{0}\left(p_{n+1}-1\right)}=\frac{1-\theta}{p_{n+1}}+\frac{\theta}{s_{n}} .
$$

Note that $\frac{p_{0}\left(p_{n+1}-1\right)}{p_{0}-1}>p_{n+1}$ is always satisfied provided that $p_{n+1}>p_{0}$, i.e. that the sequence $p_{n}$ is strictly monotone increasing. 
It then follows from (22) (by neglecting the second term on the left-hand side) that

$$
\frac{\mathrm{d}}{\mathrm{d} t}\|u\|_{p_{n+1}}^{p_{n+1}} \leq p_{n+1}\|f\|_{p_{0}}\|u\|_{s_{n}}^{\theta\left(p_{n+1}-1\right)}\left(\|u\|_{p_{n+1}}^{p_{n+1}}\right)^{1-\frac{1+\theta\left(p_{n+1}-1\right)}{p_{n+1}}}
$$

By applying again the elementary inequality (12) with $y(t)=\|u(t)\|_{p_{n+1}}^{p_{n+1}}$ and $r=$ $\frac{1+\theta\left(p_{n+1}-1\right)}{p_{n+1}}<1$, it yields

$$
\begin{aligned}
\|u(T)\|_{p_{n+1}}^{p_{n+1}} & \\
\leq & {\left[\left\|u_{0}\right\|_{p_{n+1}}^{1+\theta\left(p_{n+1}-1\right)}+\left(1+\theta\left(p_{n+1}-1\right)\right) \int_{0}^{T}\|f\|_{p_{0}}\|u\|_{S_{n}}^{\theta\left(p_{n+1}-1\right)} \mathrm{d} t\right]^{\frac{p_{n+1}}{1+\theta\left(p_{n+1}-1\right)}} } \\
\leq & {\left[\left\|u_{0}\right\|_{p_{n+1}}^{1+\theta\left(p_{n+1}-1\right)}+\left(1+\theta\left(p_{n+1}-1\right)\right)\|f\|_{L^{p_{0}}\left(Q_{T}\right)}\right.} \\
& \left.\left(\int_{0}^{T}\|u\|_{S_{n}}^{\theta\left(p_{n+1}-1\right) \frac{p_{0}}{p_{0}-1}} \mathrm{~d} t\right)^{\frac{p_{0}-1}{p_{0}}}\right]^{\frac{p_{n+1}}{1+\theta\left(p_{n+1}-1\right)}}
\end{aligned}
$$

In order to continue estimating by using (21), we choose $p_{n+1}$ as

$$
\theta\left(p_{n+1}-1\right) \frac{p_{0}}{p_{0}-1}=r_{n}
$$

Since $r_{n}=s_{n} \frac{d-2}{d}$, Eq. (25) implies $\frac{\theta}{s_{n}}=\left(1-\frac{2}{d}\right) \frac{p_{0}-1}{p_{0}\left(p_{n+1}-1\right)}$ and thus with (23)

$$
\theta=1-\frac{2}{d} \frac{p_{0}-1}{p_{0}} \frac{p_{n+1}}{p_{n+1}-1}<1
$$

In order to verify that above choice of $p_{n+1}$ satisfies $\frac{p_{0}\left(p_{n+1}-1\right)}{p_{0}-1}<s_{n}$, we insert (26) into (25) and calculate

$$
\begin{aligned}
& \left(p_{n+1}-1\right) \frac{p_{0}}{p_{0}-1}-\frac{2}{d} p_{n+1}=s_{n} \frac{d-2}{d} \\
& \Rightarrow \quad s_{n}-\frac{p_{0}\left(p_{n+1}-1\right)}{p_{0}-1}=\frac{2}{d}\left(s_{n}-p_{n+1}\right)>0 .
\end{aligned}
$$

Similar, by recalling $s_{n} \frac{d-2}{d}=r_{n}=m-1+p_{n}$, we get the iteration

$$
p_{n+1}=p_{n} \frac{d\left(p_{0}-1\right)}{p_{0}(d-2)+2}+\frac{d\left[(m-1)\left(p_{0}-1\right)+p_{0}\right]}{p_{0}(d-2)+2} .
$$


Altogether, by inserting (25) into (24), we obtain thanks to (21)

$$
\begin{aligned}
\|u(T)\|_{p_{n+1}}^{p_{n+1}} \leq & {\left[\left\|u_{0}\right\|_{p_{n+1}}^{1+\theta\left(p_{n+1}-1\right)}+\left(1+\theta\left(p_{n+1}-1\right)\right)\right.} \\
& \left.\|f\|_{L^{p_{0}\left(Q_{T}\right)}}\left(\int_{0}^{T}\|u\|_{S_{n}}^{r_{n}} \mathrm{~d} t\right)^{\frac{p_{0}-1}{p_{0}}}\right]^{\frac{p_{n+1}}{1+\theta\left(p_{n+1}-1\right)}} \\
\leq & {\left[\left\|u_{0}\right\|_{p_{n+1}}^{1+\theta\left(p_{n+1}-1\right)}+\left(1+\theta\left(p_{n+1}-1\right)\right)\right.} \\
& \left.\|f\|_{L^{p_{0}\left(Q_{T}\right)}} D_{T, n}^{\frac{p_{0}-1}{p_{0}}}\right]^{\frac{p_{n+1}}{1+\theta\left(p_{n+1}-1\right)}}=: C_{T, n+1}
\end{aligned}
$$

and thus

$$
u \in L^{\infty}\left(0, T ; L^{p_{n+1}}(\Omega)\right) \quad \text { and } \quad\|u(T)\|_{p_{n+1}}^{p_{n+1}} \leq C_{T, n+1} .
$$

Step 4 (Iteration of (21)). We will use similar arguments to Step 2. Integrating (22) and adding $\left.\left.\int_{0}^{T} \int_{\Omega}|| u\right|^{\frac{r_{n+1}}{2}}\right|^{2} \mathrm{~d} x \mathrm{~d} t$ to both sides yields in particular

$$
\begin{aligned}
& C\left(p_{n+1}\right) \int_{0}^{T}\left\||u|^{\frac{r_{n+1}}{2}}\right\|_{H^{1}(\Omega)}^{2} \mathrm{~d} t=C\left(p_{n+1}\right) \int_{0}^{T} \int_{\Omega}\left[\left|\nabla\left(|u|^{\frac{r_{n+1}}{2}}\right)\right|^{2} \mathrm{~d} x\right. \\
& \left.+\left.\left.|| u\right|^{\frac{r_{n+1}}{2}}\right|^{2} \mathrm{~d} x\right] \mathrm{d} t \\
& \leq\left\|u_{0}\right\|_{p_{n+1}}^{p_{n+1}}+p_{n+1} \int_{0}^{T}\|f\|_{p_{0}}\|u\|_{\frac{p_{0}\left(p_{n+1}-1\right)}{p_{0}-1}}^{p_{n+1}-1} \mathrm{~d} t \\
& +C\left(p_{n+1}\right) \int_{0}^{T}\|u\|_{r_{n+1}}^{r_{n+1}} \mathrm{~d} t \\
& \leq\left\|u_{0}\right\|_{p_{n+1}}^{p_{n+1}}+p_{n+1} \int_{0}^{T}\|f\|_{p_{0}}\|u\|_{S_{n}}^{\theta\left(p_{n+1}-1\right)}\|u\|_{p_{n+1}}^{(1-\theta)\left(p_{n+1}-1\right)} \mathrm{d} t \\
& +C\left(p_{n+1}\right) \int_{0}^{T}\|u\|_{r_{n+1}}^{r_{n+1}} \mathrm{~d} t \quad(\theta \text { in }(23)) \\
& \leq\left\|u_{0}\right\|_{p_{n+1}}^{p_{n+1}}+p_{n+1} C_{T, n+1}^{(1-\theta) \frac{\left(p_{n+1}-1\right)}{p_{n+1}}} \int_{0}^{T}\|f\|_{p_{0}}\|u\|_{s_{n}}^{\theta\left(p_{n+1}-1\right)} \mathrm{d} t \\
& +C\left(p_{n+1}\right) \int_{0}^{T}\|u\|_{r_{n+1}}^{r_{n+1}} \mathrm{~d} t \quad \text { (using (29)) } \\
& \leq\left\|u_{0}\right\|_{p_{n+1}}^{p_{n+1}}+p_{n+1} C_{T, n+1}^{(1-\theta) \frac{\left(p_{n+1}-1\right)}{p_{n+1}}}\|f\|_{L^{p_{0}\left(Q_{T}\right)}}\left(\int_{0}^{T}\|u\|_{S_{n}}^{r_{n}} \mathrm{~d} t\right)^{\frac{p_{0}-1}{p_{0}}} \\
& +C\left(p_{n+1}\right) \int_{0}^{T}\|u\|_{r_{n+1}}^{r_{n+1}} \mathrm{~d} t \quad \text { (using (25)) }
\end{aligned}
$$




$$
\begin{aligned}
\leq & \left\|u_{0}\right\|_{p_{n+1}}^{p_{n+1}}+p_{n+1} C_{T, n+1}^{(1-\theta) \frac{\left(p_{n+1}-1\right)}{p_{n+1}}}\|f\|_{L^{p_{0}}\left(Q_{T}\right)} D_{T, n}^{\frac{p_{0}-1}{p_{0}}} \\
& +C\left(p_{n+1}\right) \int_{0}^{T}\|u\|_{r_{n+1}}^{r_{n+1}} \mathrm{~d} t \quad \text { (using (21)). }
\end{aligned}
$$

Now by Sobolev's embedding

$$
\begin{gathered}
C\left(p_{n+1}\right) \int_{0}^{T}\left\||u|^{\frac{r_{n+1}}{2}}\right\|_{H^{1}(\Omega)}^{2} \mathrm{~d} t \geq C\left(p_{n+1}\right) C_{S}^{2} \int_{0}^{T}\|u\|_{s_{n+1}}^{r_{n+1}} \mathrm{~d} t \\
\text { with } \quad s_{n+1}= \begin{cases}\frac{r_{n+1} d}{d-2} & \text { if } d \geq 3, \\
r_{n+1}<s_{n+1}<\infty \text { arbitrary } & \text { if } d=1,2 .\end{cases}
\end{gathered}
$$

By the bound $\|u(t)\|_{p_{n+1}}^{p_{n+1}} \leq C_{T, n+1}$, the interpolation inequality

$$
\begin{aligned}
\|u\|_{r_{n+1}} & \leq\|u\|_{p_{n+1}}^{\gamma}\|u\|_{s_{n+1}}^{1-\gamma} \leq C_{T, n+1}^{\gamma / p_{n+1}}\|u\|_{s_{n+1}}^{1-\gamma} \\
\text { with } & \frac{1}{r_{n+1}}=\frac{\gamma}{p_{n+1}}+\frac{1-\gamma}{s_{n+1}} \text { for } \gamma=\frac{2 p_{n+1}}{2 p_{n+1}+(m-1) d} \in(0,1] .
\end{aligned}
$$

Like in Step 2 in case $m>1$ and $\gamma<1$, we have by Young's inequality,

$$
\begin{aligned}
C\left(p_{n+1}\right) \int_{0}^{T}\|u\|_{r_{n+1}}^{r_{n+1}} \mathrm{~d} t & \leq C\left(p_{n+1}\right) \int_{0}^{T} C_{T, n+1}^{\gamma r_{n+1} / p_{n+1}}\|u\|_{s_{n+1}}^{(1-\gamma) r_{n+1}} \mathrm{~d} t \\
& \leq \frac{C\left(p_{n+1}\right) C_{S}^{2}}{2} \int_{0}^{T}\|u\|_{s_{n+1}}^{r_{n+1}} \mathrm{~d} t+C T C_{T, n+1}^{r_{n+1} / p_{n+1}}
\end{aligned}
$$

analogue to (17) while the case $m=1$ and $r_{n+1} / p_{n+1}=1$ follows without interpolation and the first term on the right-hand side above. Combining (30), (31) and (32) yields

$$
\begin{aligned}
\frac{C\left(p_{n+1}\right) C_{S}^{2}}{2} \int_{0}^{T}\|u\|_{S_{n+1}}^{r_{n+1}} \mathrm{~d} t \leq & \left\|u_{0}\right\|_{p_{n+1}}^{p_{n+1}}+p_{n+1} C_{T, n+1}^{(1-\theta) \frac{\left(p_{n+1}-1\right)}{p_{n+1}}}\|f\|_{L^{p_{0}}\left(Q_{T}\right)} D_{T, n}^{\frac{p_{0}-1}{p_{0}}} \\
& +C T C_{T, n+1}^{r_{n+1} / p_{n+1}}
\end{aligned}
$$

hence

$$
\int_{0}^{T}\|u\|_{S_{n+1}}^{r_{n+1}} \mathrm{~d} t \leq D_{T, n+1}
$$

with

$$
\begin{aligned}
D_{T, n+1}:= & \frac{2}{C\left(p_{n+1}\right) C_{S}^{2}}\left[\left\|u_{0}\right\|_{p_{n+1}}^{p_{n+1}}+p_{n+1} C_{T, n+1}^{(1-\theta) \frac{\left(p_{n+1}-1\right)}{p_{n+1}}}\|f\|_{L^{p_{0}\left(Q_{T}\right)}} D_{T, n}^{\frac{p_{0}-1}{p_{0}}}\right. \\
& \left.+C T C_{T, n+1}^{r_{n+1} / p_{n+1}}\right] .
\end{aligned}
$$


Step 5. Passing to the limit as $n \rightarrow \infty$. Considering the iteration (27), the only possible fixed point $p_{\infty}$ of the sequence $p_{n}$ is

$$
p_{\infty}=\frac{d\left[(m-1)\left(p_{0}-1\right)+p_{0}\right]}{2\left[\frac{d+2}{2}-p_{0}\right]} .
$$

Hence, $p_{\infty}<0$ if and only if $p_{0}>\frac{d+2}{2}$. In particular, it is straightforward to check that the sequence $p_{n}$ defined by (27) is strictly monotone increasing if and only if either $p_{n}<p_{\infty}$ in the case $p_{0}<\frac{d+2}{2}$ or $p_{n}>p_{\infty}$ in the case $p_{0}>\frac{d+2}{2}$ when $p_{\infty}<0$ holds or $p_{0}=\frac{d+2}{2}$ where $p_{\infty}=+\infty$.

Therefore, we have as $n \rightarrow \infty$

$$
p_{n} \longrightarrow\left\{\begin{array}{lll}
p_{\infty} & \text { if } & p_{0}<\frac{d+2}{2} \\
+\infty & \text { if } & p_{0} \geq \frac{d+2}{2}
\end{array}\right.
$$

Step 6 (Interpolation). From (20) and (21) and by using the interpolation

$$
L^{\infty}\left(0, T ; L^{p_{n}}(\Omega)\right) \cap L^{r_{n}}\left(0, T ; L^{S_{n}}(\Omega)\right) \hookrightarrow L^{\frac{d+2}{d} p_{n}+m-1}\left(Q_{T}\right)
$$

we get $u \in L^{r}\left(Q_{T}\right)$ for all $r<\infty$ in the case $p_{0} \geq \frac{d+2}{2}$. In the case $p_{0}<\frac{d+2}{2}$, we obtain $u \in L^{s}\left(Q_{T}\right)$ for all

$$
s<\frac{d+2}{d} p_{\infty}+m-1=\frac{(m d+2) p_{0}}{d+2-2 p_{0}} .
$$

This completes the proof of Lemma 2.1.

Lemma 2.2. Let $u$ be a weak solution to $(\mathrm{S})$ and

$$
\|u\|_{L^{q_{0}}\left(Q_{T}\right)} \leq C_{T}, \quad \forall i=1, \ldots, S, \quad \text { with } q_{0}>\frac{d(v-m)+2(v-1)}{2}
$$

where $m=\min \left\{m_{i}: i=1 \ldots S\right\}, v$ is defined in $(\mathrm{G})$, and $C_{T}$ is growing at most polynomially in $T$.

Then, it follows that $\left\|u_{i}\right\|_{L^{\infty}\left(Q_{T}\right)} \leq C_{T}$ for all $i=1 \ldots S$.

Proof. From $u_{i} \in L^{q_{0}}\left(Q_{T}\right)$ for all $i=1, \ldots, S$, we have $f_{i}(u) \in L^{q_{0} / v}\left(Q_{T}\right)$. Moreover, note that the quasi-positivity assumption $(\mathrm{P})$ ensures non-negative solutions $u$ for non-negative initial data $u_{i, 0}$. Hence, the concentrations $u_{i}$ satisfy the (non-signchanging) porous media equation

$$
\partial_{t} u_{i}-d_{i} \Delta\left(u_{i}^{m_{i}}\right)=f_{i}(u) \in L^{q_{0} / v}\left(Q_{T}\right) .
$$

Lemma 2.1 implies that if $q_{0} / v \geq \frac{d+2}{2}$, then $u_{i} \in L^{r}\left(Q_{T}\right)$ for all $r<\infty$, while if $q_{0} / v<\frac{d+2}{2}$, then 


$$
\begin{aligned}
u_{i} & \in L^{s}\left(Q_{T}\right) \quad \text { for all } \quad s<q_{1}:=\frac{(m d+2) q_{0}}{v(d+2)-2 q_{0}} \\
\leq & \frac{\left(m_{i} d+2\right) q_{0}}{v(d+2)-2 q_{0}}, \quad \text { for all } i=1 \ldots S,
\end{aligned}
$$

since $m \leq m_{i}$. We then construct a sequence $q_{n}$ (equally for all $i=1, \ldots, S$ ) such that

$$
q_{n+1}=\frac{(m d+2) q_{n}}{v(d+2)-2 q_{n}} \quad \text { for } n \geq 0 .
$$

It follows that

$$
\frac{q_{n+1}}{q_{n}}=\frac{m d+2}{v(d+2)-2 q_{n}} .
$$

Therefore, as long as $v(d+2)-2 q_{n}>0 \Longleftrightarrow q_{n}<\frac{(d+2) v}{2}$,

$$
\frac{q_{n+1}}{q_{n}}>1 \text { for all } n \geq 0 \quad \Longleftrightarrow \quad q_{0}>\frac{d(v-m)+2(v-1)}{2} \text {. }
$$

Hence with $q_{0}>\frac{d(v-m)+2(v-1)}{2}$, after finitely many steps, we arrive at $q_{n}>\frac{(d+2) v}{2}$. From $u_{i} \in L^{S}\left(Q_{T}\right)$ for all $s<q_{n}$, we have in particular $u_{i} \in L^{\frac{(d+2) v}{2}}\left(Q_{T}\right)$, which implies $f_{i}(u) \in L^{\frac{d+2}{2}}\left(Q_{T}\right)$ for $i=1, \ldots, S$. By applying Lemma 2.1 once more, we obtain $u_{i} \in L^{r}\left(Q_{T}\right)$ for all $r, q<\infty$. Thus,

$$
\partial_{t} u_{i}-d_{i} \Delta\left(u_{i}^{m_{i}}\right)=f_{i}(u) \in L^{r}\left(Q_{T}\right) \text { for all } s<\infty
$$

with $\left\|f_{i}(u)\right\|_{L^{r}\left(Q_{T}\right)} \leq C_{T}$ for some $r>\frac{d+2}{2}$. Therefore,

$$
\left\|u_{i}\right\|_{L^{\infty}\left(Q_{T}\right)} \leq C_{T} \quad \text { for all } \quad i=1, \ldots, S,
$$

thanks to the following Lemma 2.3.

Lemma 2.3. Let $u$ be the solution to

$$
\begin{cases}\partial_{t} u-\delta \Delta\left(|u|^{m-1} u\right)=f, & (x, t) \in Q_{T}, \\ \nabla\left(|u|^{m-1} u\right) \cdot \vec{n}=0, & (x, t) \in \partial \Omega \times(0, T), \\ u(x, 0)=u_{0}(x), & x \in \Omega,\end{cases}
$$

with $u_{0} \in L^{\infty}(\Omega)$ and $\|f\|_{L^{q}\left(Q_{T}\right)} \leq C_{T}$ for some $q>\frac{d+2}{2}$. Then,

$$
\|u\|_{L^{\infty}\left(Q_{T}\right)} \leq C_{T} .
$$

Though the boundedness result of this Lemma has been cited in many works, we are unable to find a precise reference. We therefore give in this paper a full proof based on the famous Moser iteration. Moreover, our proof shows the polynomial growth of the $L^{\infty}$-norm in (35), which is important for our sequel analysis.

To prove Lemma 2.3, we need the following two lemmas. 
Lemma 2.4. [7, Lemma 2.5] Let $\left\{y_{n}\right\}_{n \geq 1}$ be a sequence of positive numbers which satisfies

$$
y_{n+1} \leq K B^{n}\left(y_{n}^{\gamma}+y_{n}^{\kappa}\right)
$$

where $K, B>0$ and $\gamma, \kappa>1$ are independent of $n$. Then there exists $\varepsilon>0$ such that, if $y_{1} \leq \varepsilon$, then

$$
\lim _{n \rightarrow \infty} y_{n}=0
$$

Lemma 2.5. [27, II.§3] Define

$$
\begin{aligned}
W(0, T):= & \left\{u: Q_{T} \rightarrow \mathbb{R} \text { such that }\|u\|_{W(0, T)}^{2}:=\sup _{t \in(0, T)}\|u(t)\|^{2}\right. \\
& \left.+\int_{0}^{T}\|u(t)\|_{H^{1}(\Omega)}^{2} d t<+\infty\right\}
\end{aligned}
$$

For $p, q$ satisfying

$$
\frac{1}{p}+\frac{d}{2 q}=\frac{d}{4}
$$

there exists a constant $C$ independent of $T$ such that

$$
\|u\|_{L^{p}\left(0, T ; L^{q}(\Omega)\right)} \leq C\|u\|_{W(0, T)} .
$$

In particular, when $p=q=2+\frac{4}{d}$,

$$
\|u\|_{L^{2+\frac{4}{d}\left(Q_{T}\right)}} \leq C\|u\|_{W(0, T)} .
$$

Proof of Lemma 2.3. Let $k \geq 1$ be a constant which will be specified later. For each $i \geq 0$, we define

$$
v_{i}:=\left(u-k+\frac{k}{2^{i}}\right)_{+}=\max \left\{u-k+\frac{k}{2^{i}} ; 0\right\}
$$

and

$$
A_{i}:=\left\{(x, t) \in Q_{T}: u(x, t) \geq k-\frac{k}{2^{i}}\right\} .
$$

The following simple observations will be helpful

$$
\begin{aligned}
v_{i+1}(x, t) & \leq v_{i}(x, t) & & \text { for all }(x, t) \in A_{i}, \\
v_{i}(x, t) & \geq \frac{k}{2^{i+1}} & & \text { for all }(x, t) \in A_{i+1} \subset A_{i} .
\end{aligned}
$$


By multiplying the equation $\partial_{t} u-\delta \Delta\left(|u|^{m-1} u\right)=f$ by $v_{i+1}$ and integrating on $Q_{T}$, we have

$$
\begin{aligned}
& \sup _{t \in(0, T)}\left\|v_{i+1}(t)\right\|^{2}+2 \delta m \int_{0}^{T} \int_{\Omega}|u|^{m-1}\left|\nabla v_{i+1}\right|^{2} \mathrm{~d} x \mathrm{~d} t \\
& \leq\left\|v_{i+1}(0)\right\|^{2}+2 \int_{0}^{T} \int_{\Omega} f v_{i+1} \mathrm{~d} x \mathrm{~d} t .
\end{aligned}
$$

Note that $u \geq k-\frac{k}{2^{i}} \geq \frac{k}{2}$ on $A_{i}$, we have

$$
\begin{aligned}
2 \delta m \int_{0}^{T} \int_{\Omega}|u|^{m-1}\left|\nabla v_{i+1}\right|^{2} \mathrm{~d} x & \geq 2 \delta m \iint_{A_{i}}|u|^{m-1}\left|\nabla v_{i+1}\right|^{2} \mathrm{~d} x \\
& \geq \delta m \frac{k^{m-1}}{2^{m-2}} \iint_{A_{i}}\left|\nabla v_{i+1}\right|^{2} \mathrm{~d} x \mathrm{~d} t \\
& \geq \frac{\delta m}{2^{m-2}} \int_{0}^{T} \int_{\Omega}\left|\nabla v_{i+1}\right|^{2} \mathrm{~d} x \mathrm{~d} t
\end{aligned}
$$

thanks to $k \geq 1$, and the fact that $v_{i+1} \equiv 0$ on $Q_{T} \backslash A_{i+1} \supset Q_{T} \backslash A_{i}$ since $A_{i+1} \subset A_{i}$. By adding $\int_{0}^{T}\left\|v_{i+1}\right\|^{2} \mathrm{~d} t$ to both sides of (37), we get

$$
\begin{aligned}
& \sup _{t \in(0, T)}\left\|v_{i+1}(t)\right\|^{2}+\frac{\delta m}{2^{m-2}} \int_{0}^{T}\left\|v_{i+1}\right\|_{H^{1}(\Omega)}^{2} \mathrm{~d} x \mathrm{~d} t \\
& \leq C \int_{0}^{T}\left\|v_{i+1}\right\|^{2} \mathrm{~d} t+\left\|v_{i+1}(0)\right\|^{2}+\int_{0}^{T} \int_{\Omega} f v_{i+1} \mathrm{~d} x \mathrm{~d} t .
\end{aligned}
$$

which yields

$$
C\left\|v_{i+1}\right\|_{W(0, T)}^{2} \leq \int_{0}^{T}\left\|v_{i+1}\right\|^{2} \mathrm{~d} t+\left\|v_{i+1}(0)\right\|^{2}+\int_{0}^{T} \int_{\Omega} f v_{i+1} \mathrm{~d} x \mathrm{~d} t .
$$

By definition,

$$
\left\|v_{i+1}(0)\right\|^{2}=\left\|\left(u_{0}-k+\frac{k}{2^{i+1}}\right)_{+}\right\|^{2}=0
$$

when we choose $k \geq 2\left\|u_{0}\right\|_{L^{\infty}(\Omega)}$. By using (36), we have with $1 \leq \frac{2^{i+1}}{k} v_{i}$ on $A_{i+1}$

$$
\begin{aligned}
\int_{0}^{T} \int_{\Omega}\left|v_{i+1}\right|^{2} \mathrm{~d} x \mathrm{~d} t & =\int_{0}^{T} \int_{\Omega} \mathbf{1}_{A_{i+1}}\left|v_{i+1}\right|^{2} \mathrm{~d} x \mathrm{~d} t \\
& \leq \int_{0}^{T} \int_{\Omega} \mathbf{1}_{A_{i+1}}\left|v_{i}\right|^{2} \mathrm{~d} x \mathrm{~d} t \\
& \leq\left(\frac{2^{i+1}}{k}\right)^{\frac{4}{d}} \int_{0}^{T} \int_{\Omega} \mathbf{1}_{A_{i+1}}\left|v_{i}\right|^{2+\frac{4}{d}} \mathrm{~d} x \mathrm{~d} t \\
& \leq C\left(2^{4 / d}\right)^{i}\left\|v_{i}\right\|_{W(0, T)}^{2+\frac{4}{d}} .
\end{aligned}
$$


Since $q>\frac{d+2}{2}$, we have

$$
\sigma:=\frac{q-1}{q}\left(2+\frac{4}{d}\right)>2
$$

Moreover,

$$
\frac{\sigma q}{q-1}=2+\frac{4}{d}
$$

thus

$$
\left\|v_{i}\right\|_{L^{\frac{\sigma q}{q-1}}\left(Q_{T}\right)} \leq C\left\|v_{i}\right\|_{W(0, T)} .
$$

We now can use Hölder's inequality to estimate with (36)

$$
\begin{aligned}
\int_{0}^{T} \int_{\Omega} f v_{i+1} \mathrm{~d} x \mathrm{~d} t & \leq \int_{0}^{T} \int_{\Omega} f v_{i+1}\left(\frac{2^{i+1}}{k}\right)^{\sigma-1} v_{i}^{\sigma-1} \mathrm{~d} x \mathrm{~d} t \\
& \leq\left(\frac{2^{i+1}}{k}\right)^{\sigma-1} \int_{0}^{T} \int_{\Omega}\left|f \| v_{i}\right|^{\sigma} \mathrm{d} x \mathrm{~d} t \\
& \leq C\left(2^{\sigma-1}\right)^{i}\|f\|_{L^{q}\left(Q_{T}\right)}\left\|v_{i}\right\|_{L^{\frac{\sigma q}{q-1}}\left(Q_{T}\right)}^{\sigma} \\
& \leq C\left(2^{\sigma-1}\right)^{i}\|f\|_{L^{q}\left(Q_{T}\right)}\left\|v_{i}\right\|_{W(0, T)}^{\sigma} .
\end{aligned}
$$

Inserting (38), (39) and (41) into (37) leads to

$$
\left\|v_{i+1}\right\|_{W(0, T)}^{2} \leq C\left(1+\|f\|_{L^{q}\left(Q_{T}\right)}\right) B^{i}\left(\left\|v_{i}\right\|_{W(0, T)}^{2+\frac{4}{d}}+\left\|v_{i}\right\|_{W(0, T)}^{\sigma}\right)
$$

for all $i \geq 0$, where $B=\max \left\{2^{4 / d} ; 2^{\sigma-1}\right\}$. By setting $Y_{i}=\left\|v_{i}\right\|_{W(0, T)}^{2}$, we obtain a sequence $\left\{Y_{n}\right\}_{n \geq 1}$ satisfying the property in Lemma 2.4. It remains to show that $Y_{1}$ is small enough.

We show now that for any $\varepsilon>0$, there exists $k \geq \max \left\{1 ; 2\left\|u_{0}\right\|_{L^{\infty}(\Omega)}\right\}$ large enough such that

$$
Y_{1}=\left\|v_{1}\right\|_{W(0, T)} \leq \varepsilon
$$

From Step 2 in the proof of Lemma 2.1, we have

$$
\|u\|_{L^{\infty}\left(0, T ; L^{q}(\Omega)\right)}+\|u\|_{L^{r}\left(0, T ; L^{s}(\Omega)\right)} \leq C_{T}
$$

where $r=m+q-1 \geq q$ and $s=\frac{r d}{d-2}$ if $d \geq 2$ and $r<s<+\infty$ arbitrary if $d \leq 2$. By interpolation, see e.g. [15, Lemma 4.1], we see that

$$
\|u\|_{L^{\tau}\left(Q_{T}\right) \leq C_{T}} \quad \text { with } \quad \tau= \begin{cases}\frac{d r+2 q}{d} & \text { if } d \geq 3 \\ <r+q \text { arbitrary } & \text { if } d \leq 2\end{cases}
$$


Direct calculations show that $\tau>2+\frac{4}{d}$ if $d \geq 2$ and $\tau>3$ if $d=1$. In particular,

$$
\|u\|_{L^{2+\frac{4}{d}}\left(Q_{T}\right)} \leq C_{T} \text { for } d \geq 2 \quad \text { and } \quad\|u\|_{L^{3}\left(Q_{T}\right)} \leq C_{T} \text { for } d=1 .
$$

From (38),

$$
C\left\|v_{1}\right\|_{W(0, T)}^{2} \leq \int_{0}^{T}\left\|v_{1}(t)\right\|^{2} \mathrm{~d} t+\left\|v_{1}(0)\right\|^{2}+\int_{0}^{T} \int_{\Omega} f v_{1} \mathrm{~d} x \mathrm{~d} t .
$$

Since $k \geq 2\left\|u_{0}\right\|_{L^{\infty}(\Omega)},\left\|v_{1}(0)\right\|^{2}=\left\|\left(u_{0}-k / 2\right)_{+}\right\|^{2}=0$.

Consider now the case $d \geq 2$. By using (36), it yields

$$
\begin{aligned}
\int_{0}^{T} \int_{\Omega}\left|v_{1}\right|^{2} \mathrm{~d} x \mathrm{~d} t & =\int_{0}^{T} \int_{\Omega} \mathbf{1}_{A_{1}}\left|v_{1}\right|^{2} \mathrm{~d} x \mathrm{~d} t \leq\left(\frac{4}{k}\right)^{\frac{4}{d}} \int_{0}^{T} \int_{\Omega}\left|v_{0}\right|^{2+\frac{4}{d}} \mathrm{~d} x \mathrm{~d} t \\
& \leq\left(\frac{4}{k}\right)^{\frac{4}{d}}\|u\|_{L^{2+\frac{4}{d}}}^{2+\frac{4}{d}} \leq\left(\frac{4}{k}\right)^{\frac{4}{d}} C_{T},
\end{aligned}
$$

recalling that $v_{0}=u_{+}$. Similarly to (41), we get

$$
\int_{0}^{T} \int_{\Omega} f v_{1} \mathrm{~d} x \mathrm{~d} t \leq\left(\frac{4}{k}\right)^{\sigma-1}\|f\|_{L^{q}\left(Q_{T}\right)}\|u\|_{L^{2+\frac{4}{d}\left(Q_{T}\right)}}^{\sigma} \leq\left(\frac{4}{k}\right)^{\sigma-1} C_{T} .
$$

From (42), (45) and (46), we get (43) if

$$
k=4 \max \left\{\left(\frac{C_{T}}{\varepsilon}\right)^{\frac{d}{4}} ;\left(\frac{C_{T}}{\varepsilon}\right)^{\frac{1}{\sigma-1}}\right\} .
$$

Thus, with this choice of $k$, it follows that

$$
0=\lim _{i \rightarrow \infty} Y_{i}=\left\|(u-k)_{+}\right\|^{2},
$$

and hence,

$$
\|u\|_{L^{\infty}\left(Q_{T}\right)} \leq k=4 \max \left\{\left(\frac{C_{T}}{\varepsilon}\right)^{\frac{d}{4}} ;\left(\frac{C_{T}}{\varepsilon}\right)^{\frac{1}{\sigma-1}}\right\}
$$

which is our desired estimate.

The proof for the case $d=1$ is very similar using the

$$
\int_{0}^{T} \int_{\Omega}\left|v_{1}\right|^{2} \mathrm{~d} x \mathrm{~d} t \leq \frac{4}{k} \int_{0}^{T} \int_{\Omega}\left|v_{0}\right|^{3} \mathrm{~d} x \mathrm{~d} t \leq \frac{4}{k} C_{T}
$$

and

$$
\int_{0}^{T} \int_{\Omega} f v_{1} \mathrm{~d} x \mathrm{~d} t \leq\left(\frac{4}{k}\right)^{\frac{4 \xi}{1+2 \xi}}\|f\|_{L^{q}\left(Q_{T}\right)}\|u\|_{L^{3}\left(Q_{T}\right)}^{1+\frac{4 \xi}{1+2 \xi}} \leq\left(\frac{4}{k}\right)^{\frac{4 \xi}{1+2 \xi}} C_{T}
$$

where $\xi=\frac{1}{2}(2 q-3)>0$. We therefore omit the details. 
Now, we are ready to prove the boundedness of solutions to (S):

Proof of Theorem 1.2. Assuming $m_{i}>v-1$, the existence of weak solutions follows similar to $[26,38]$ and is proven in Sect. 5 in detail. By the duality estimates in Lemma 5.1, we have

$$
u_{i} \in L^{m_{i}+1}\left(Q_{T}\right) \text { for all } i=1, \ldots, S
$$

Because $m_{i}>v-\frac{4}{2+d}$, it follows that

$$
m_{i}+1>\frac{d\left(v-m_{i}\right)+2(v-1)}{2} .
$$

Therefore, Lemma 2.2 yields $u_{i} \in L^{\infty}\left(Q_{T}\right)$ and $\left\|u_{i}\right\|_{L^{\infty}\left(Q_{T}\right)} \leq C_{T}$ for arbitrary $T>0$, which shows that the weak solutions are bounded and the $L^{\infty}(\Omega)$ norms grows at most polynomially in time.

The local Hölder continuity of the bounded weak solutions is a classical result, see e.g. [9] or [43, Theorem 7.18].

\section{Convergence to equilibrium}

In this section, we prove exponential convergence to equilibrium of solutions to $(\mathrm{R})$ by using the entropy method. We start by recalling the entropy (free energy) functional

$$
E[a, b]=\sum_{i=1}^{M} \int_{\Omega}\left(a_{i} \ln a_{i}-a_{i}+1\right) \mathrm{d} x+\sum_{j=1}^{N} \int_{\Omega}\left(b_{j} \ln b_{j}-b_{j}+1\right) \mathrm{d} x
$$

and its non-negative entropy production (free energy dissipation) functional $D[a, b]:=$ $-\frac{\mathrm{d}}{\mathrm{d} t} E[a, b]$, i.e.

$$
D[a, b]=\sum_{i=1}^{M} d_{i} \int_{\Omega} \frac{\left|\nabla a_{i}\right|^{2}}{a_{i}^{2-m_{i}}} \mathrm{~d} x+\sum_{j=1}^{N} h_{j} \int_{\Omega} \frac{\left|\nabla b_{j}\right|^{2}}{b_{j}^{2-p_{j}}} \mathrm{~d} x+\int_{\Omega}\left(a^{\alpha}-b^{\beta}\right) \ln \frac{a^{\alpha}}{b^{\beta}} \mathrm{d} x \geq 0
$$

where we have used the short-hand notation

$$
a^{\alpha}=\prod_{i=1}^{M} a_{i}^{\alpha_{i}} \quad \text { and } \quad b^{\beta}=\prod_{j=1}^{N} b_{j}^{\beta_{j}} .
$$

Moreover, the following additivity property of the relative entropy holds

$$
\begin{aligned}
& E[a, b]-E\left[a_{\infty}, b_{\infty}\right] \\
& \quad=\sum_{i=1}^{M} \int_{\Omega}\left(a_{i} \ln \frac{a_{i}}{a_{i \infty}}-a_{i}+a_{i \infty}\right) \mathrm{d} x+\sum_{j=1}^{N} \int_{\Omega}\left(b_{j} \ln \frac{b_{j}}{b_{j \infty}}-b_{j}+b_{j \infty}\right) \mathrm{d} x
\end{aligned}
$$




$$
\begin{aligned}
= & \sum_{i=1}^{M} \int_{\Omega}\left(a_{i} \ln \frac{a_{i}}{\overline{a_{i}}}\right) \mathrm{d} x+\sum_{j=1}^{N} \int_{\Omega}\left(b_{j} \ln \frac{b_{j}}{\overline{b_{j}}}\right) \mathrm{d} x \\
& +\sum_{i=1}^{M} \int_{\Omega}\left(\overline{a_{i}} \ln \frac{\overline{a_{i}}}{a_{i \infty}}-\overline{a_{i}}+a_{i \infty}\right) \mathrm{d} x+\sum_{j=1}^{N} \int_{\Omega}\left(\overline{b_{j}} \ln \frac{\overline{b_{j}}}{b_{j \infty}}-\overline{b_{j}}+b_{j \infty}\right) \mathrm{d} x .
\end{aligned}
$$

The first Lemma 3.1 of this section states the generalisation of the logarithmic Sobolev inequality, which shall use in our approach.

Lemma 3.1. (A generalised logarithmic Sobolev inequalities, [34]) Assume that $m \geq$ $(d-2)_{+} / d$ where $(d-2)_{+}=\max \{0, d-2\}$. Then, there exists a constant $C(\Omega, m)>0$ such that

$$
\int_{\Omega} \frac{|\nabla u|^{2}}{u^{2-m}} d x \geq C(\Omega, m) \bar{u}^{m-1} \int_{\Omega} u \ln \frac{u}{\bar{u}} d x \geq C(\Omega, m) \bar{u}^{m-1}\|\sqrt{u}-\overline{\sqrt{u}}\|^{2}
$$

where $\bar{u}=\int_{\Omega} u d x$.

Proof. The first inequality follows from [34]. The second estimate follows from an elementary inequality:

$$
\int_{\Omega} u \ln \frac{u}{\bar{u}} \mathrm{~d} x=\int_{\Omega}\left(u \ln \frac{u}{\bar{u}}-u+\bar{u}\right) \mathrm{d} x \geq \int_{\Omega}(\sqrt{u}-\sqrt{\bar{u}})^{2} \mathrm{~d} x .
$$

The estimates in Lemma 3.1 constitute a generalisation of the logarithmic Sobolev inequality (5), which is recovered by setting $m=1$ and for which the pre-factor $\bar{u}^{m-1}$ vanishes. In the case of porous media diffusion $m>1$, the pre-factor $\bar{u}^{m-1}$ causes the lower bounds in Lemma 3.1 to degenerate for small spatial averages $\bar{u}$. In particular, we have by Lemma 3.1 the following lower bound for the entropy production

$$
\begin{aligned}
D[a, b] \geq & \sum_{i=1}^{M} d_{i} C\left(\Omega, m_{i}\right) \overline{a_{i}} m_{i}-1 \int_{\Omega} a_{i} \ln \frac{a_{i}}{\overline{a_{i}}} \mathrm{~d} x \\
& +\sum_{j=1}^{N} h_{j} C\left(\Omega, p_{j}\right) \overline{b_{j}} p_{j}-1 \int_{\Omega} b_{j} \ln \frac{b_{j}}{\overline{b_{j}}} \mathrm{~d} x+\int_{\Omega}\left(a^{\alpha}-b^{\beta}\right) \ln \frac{a^{\alpha}}{b^{\beta}} \mathrm{d} x \\
\geq & C_{0}\left[\sum_{i=1}^{M} \overline{a_{i}} m_{i}-1 \int_{\Omega} a_{i} \ln \frac{a_{i}}{\overline{a_{i}}} \mathrm{~d} x\right. \\
& \left.+\sum_{j=1}^{N} \overline{b_{j}} p_{j}-1 \int_{\Omega} b_{j} \ln \frac{b_{j}}{\overline{b_{j}}} \mathrm{~d} x+\int_{\Omega}\left(a^{\alpha}-b^{\beta}\right) \ln \frac{a^{\alpha}}{b^{\beta}} \mathrm{d} x\right] .
\end{aligned}
$$

The problem of degeneracy appears when some averages $\overline{a_{i}}$ or $\overline{b_{j}}$ do not satisfy a positive lower bound. To overcome this problem, we first observe that due to the 
mass conservation laws (3) not all spatial averages can be small at the same time. If, for instance, a particular $\bar{a}_{i}$ is sufficiently small (w.r.t. $M_{i j}$ ), then another $\bar{b}_{j}$ can't be arbitrarily small because of a mass conservation law (3) connecting these two species, i.e.

$$
\beta_{j} \overline{a_{i}}+\alpha_{i} \overline{b_{j}}=M_{i j}>0
$$

The following crucial Lemma 3.2 shows functional inequalities, which quantity the so-called "indirect diffusion effect" and allows to compensate the lacking lower bounds for the species, whose spatial averages do not satisfy a lower bound.

We first introduce some convenient notations:

$$
\begin{array}{lc}
A_{i}=\sqrt{a_{i}}, A_{i \infty}=\sqrt{a_{i \infty}}, & B_{j}=\sqrt{b_{j}}, B_{j \infty}=\sqrt{b_{j \infty}}, \\
\delta_{i}(x)=A_{i}(x)-\overline{A_{i}}, \quad \forall x \in \Omega, & \eta_{j}(x)=B_{j}(x)-\overline{B_{j}}, \quad \forall x \in \Omega,
\end{array}
$$

where

$$
\overline{A_{i}}=\int_{\Omega} A_{i} \mathrm{~d} x \text { and } \overline{B_{j}}=\int_{\Omega} B_{j} \mathrm{~d} x .
$$

Moreover,

$$
A^{\alpha}=\prod_{i=1}^{M} A_{i}^{\alpha_{i}} \quad \text { and } \quad B^{\beta}=\prod_{j=1}^{N} B_{j}^{\beta_{j}}
$$

The conservation laws are now rewritten as

$$
\beta_{j} \overline{A_{i}^{2}}+\alpha_{i} \overline{B_{j}^{2}}=M_{i j}>0 \quad \forall i=1 \ldots M, j=1 \ldots N
$$

Lemma 3.2. ("Indirect diffusion transfer" functional inequality) Let $A_{i}, B_{j}: \Omega \rightarrow$ $\mathbb{R}_{+}$with $i=1 \ldots M$ and $j=1 \ldots N$ be non-negative functions satisfying the conservation laws (50) and $\varepsilon>0$ be a constant to be determined later. Assume that for some $J \in\{1, \ldots, N\}$,

$$
\overline{B_{j}^{2}} \leq \varepsilon \quad \text { for all } j=1 \ldots J \text {. }
$$

Then, there exists a constant $K_{1}$ which depends on $\varepsilon$ such that:

$$
\sum_{i=1}^{M}\left\|\delta_{i}\right\|^{2}+\sum_{j=J+1}^{N}\left\|\eta_{j}\right\|^{2}+\left\|A^{\alpha}-B^{\beta}\right\|^{2} \geq K_{1} \sum_{j=1}^{J}\left\|\eta_{j}\right\|^{2}
$$

Remark 3.1. Note that when the last term on the left-hand side $\left\|A^{\alpha}-B^{\beta}\right\|^{2}$ diverges, the inequality holds trivially. Therefore, in the proof, we only consider the case when it is finite. 
Proof. Due to the mass conservation laws (50), we have the following natural bounds,

$$
\overline{A_{i}^{2}}, \overline{B_{j}^{2}} \leq M_{0}^{2}, \quad \forall i=1, \ldots, M, \forall j=1, \ldots, N
$$

for some constant $M_{0}>0$. Therefore, by Jensen's inequality, recalling that $|\Omega|=1$,

$$
\overline{A_{i}} \leq \sqrt{\overline{A_{i}^{2}}} \leq M_{0}, \quad \overline{B_{j}} \leq \sqrt{\overline{B_{j}^{2}}} \leq M_{0}, \quad \forall i, j
$$

From these bounds, we get an upper bound for the right-hand side of (51)

$$
\sum_{j=1}^{J}\left\|\eta_{j}\right\|^{2}=\sum_{j=1}^{J}\left(\overline{B_{j}^{2}}-{\overline{B_{j}}}^{2}\right) \leq \sum_{j=1}^{J} \overline{B_{j}^{2}} \leq M_{0}^{2} J
$$

We consider the following two cases.

Case 1: If there exists $i \in\{1, \ldots, M\}$ such that $\left\|\delta_{i}\right\|^{2} \geq \varepsilon$ or there exists a $j \in$ $\{J+1, \ldots, N\}$ such that $\left\|\eta_{j}\right\|^{2} \geq \varepsilon$, we have:

$$
\sum_{i=1}^{M}\left\|\delta_{i}\right\|^{2}+\sum_{j=J+1}^{N}\left\|\eta_{j}\right\|^{2}+\left\|A^{\alpha}-B^{\beta}\right\|^{2} \geq \varepsilon \geq \frac{\varepsilon}{M_{0}^{2} J} \sum_{j=1}^{J}\left\|\eta_{j}\right\|^{2}
$$

hence, the desired inequality (51) holds with $K_{1}=\frac{\varepsilon}{M_{0}^{2} J}$.

Case 2: Assume $\left\|\delta_{i}\right\|^{2} \leq \varepsilon$ for all $i \in\{1, \ldots, M\}$ and $\left\|\eta_{j}\right\|^{2} \leq \varepsilon$ for all $j \in$ $\{J+1, \ldots, N\}$, which together with the above assumption $\overline{B_{j}^{2}} \leq \varepsilon$ and $\overline{\eta_{j}^{2}} \leq \overline{B_{j}^{2}}$ for all $j=1 \ldots J$ implies $\left\|\eta_{j}\right\|^{2} \leq \varepsilon$ for all $j \in\{1, \ldots, N\}$.,

Let $\lambda>0$ and denote by

$$
\Omega_{i A}=\left\{x \in \Omega:\left|\delta_{i}(x)\right| \leq \lambda \sqrt{\varepsilon}\right\} \text { for } i=1, \ldots, M
$$

Then

$$
\varepsilon \geq \int_{\Omega}\left|\delta_{i}(x)\right|^{2} \mathrm{~d} x \geq \int_{\Omega \backslash \Omega_{i A}}\left|\delta_{i}(x)\right|^{2} \mathrm{~d} x \geq \lambda^{2} \varepsilon\left|\Omega \backslash \Omega_{i A}\right|
$$

thus

$$
\left|\Omega \backslash \Omega_{i A}\right| \leq \frac{1}{\lambda^{2}} \quad \text { which implies } \quad\left|\Omega_{i_{A}}\right| \geq 1-\frac{1}{\lambda^{2}}
$$

Similarly we get,

$$
\left|\Omega_{j B}\right| \geq 1-\frac{1}{\lambda^{2}} \quad \text { where } \Omega_{j B}=\left\{x \in \Omega:\left|\eta_{j}(x)\right| \leq \lambda \sqrt{\varepsilon}\right\} \quad \forall j=1, \ldots, N .
$$

Now choose $\lambda^{2}=2(M+N)$ and consider $G=\cap_{i=1}^{M} \Omega_{i A} \cap_{j=1}^{N} \Omega_{j B}$. Then, we have $|G| \geq \frac{1}{2}$. Note that $\left|\delta_{i}(x)\right| \leq \lambda \sqrt{\varepsilon}$ and $\left|\eta_{j}(x)\right| \leq \lambda \sqrt{\varepsilon}$ for all $x \in G$ and for all $i, j$. Moreover, $\forall x \in G$

$$
A_{i}(x)=\overline{A_{i}}+\delta_{i}(x) \leq \overline{A_{i}}+\left|\delta_{i}(x)\right| \leq M_{0}+\lambda \sqrt{\varepsilon} \leq 2 M_{0}
$$


and similarly $B_{j}(x) \leq 2 M_{0}, \forall i, j$ if we choose $\varepsilon$ such that

$$
\lambda \sqrt{\varepsilon} \leq M_{0} .
$$

By Taylor's expansion, we have

$$
A^{\alpha}=\prod_{i=1}^{M} A_{i}^{\alpha_{i}}=\prod_{i=1}^{M}\left(\overline{A_{i}}+\delta_{i}\right)^{\alpha_{i}}=\prod_{i=1}^{M}{\overline{A_{i}}}^{\alpha_{i}}+R\left(\overline{A_{i}}, \delta_{i}\right) \sum_{i=1}^{M} \delta_{i}
$$

where the remainder terms $R$ depends polynomially on $\overline{A_{i}}$ and $\delta_{i}$. Note that $\left|R\left(\bar{A}_{i}, \delta_{i}\right)\right| \leq$ $C_{0}\left(M_{0}\right)$ on $G$, we estimate with $(x-y)^{2} \geq \frac{1}{2} x^{2}-y^{2}$

$$
\begin{aligned}
\left\|A^{\alpha}-B^{\beta}\right\|^{2} & =\int_{\Omega}\left(\prod_{i=1}^{M} A_{i}^{\alpha_{i}}-B^{\beta}\right)^{2} \mathrm{~d} x \\
& \geq \int_{G}\left(\prod_{i=1}^{M} \overline{A_{i}}{ }^{\alpha_{i}}-B^{\beta}+R\left(\overline{A_{i}}, \delta_{i}\right) \sum_{i=1}^{M} \delta_{i}\right)^{2} \mathrm{~d} x \\
& \geq \frac{1}{2} \int_{G}\left(\prod_{i=1}^{M} \overline{A_{i}}{ }^{\alpha_{i}}-B^{\beta}\right)^{2} \mathrm{~d} x-\int_{G}\left|R\left(\overline{A_{i}}, \delta_{i}\right)\right|^{2}\left|\sum_{i=1}^{M} \delta_{i}\right|^{2} \\
& \geq \frac{1}{2} \int_{G}\left(\prod_{i=1}^{M} \overline{A_{i}}{ }^{\alpha_{i}}-B^{\beta}\right)^{2} \mathrm{~d} x-C_{0}\left(M_{0}\right)^{2} M \int_{G} \sum_{i=1}^{M}\left|\delta_{i}\right|^{2} \\
& \geq \frac{1}{2} \int_{G}\left(\prod_{i=1}^{M} \overline{A_{i}} \alpha_{i}-B^{\beta}\right)^{2} \mathrm{~d} x-C_{0}\left(M_{0}\right)^{2} M \int_{G} \sum_{i=1}^{M}\left\|\delta_{i}\right\|^{2} \\
& \geq \frac{1}{2} \int_{G}\left(\prod_{i=1}^{M} \overline{A_{i}} \alpha_{i}-B^{\beta}\right)^{2} \mathrm{~d} x-C_{0}\left(M_{0}\right)^{2} M^{2} \varepsilon
\end{aligned}
$$

where we used $\left\|\delta_{i}\right\|^{2} \leq \varepsilon$ in the last inequality.

In order to estimate further, we use again Taylor's expansion

$$
B^{\beta}=\prod_{j=1}^{N}\left(\overline{B_{j}}+\eta_{j}\right)^{\beta_{j}}=\prod_{j=1}^{N}{\overline{B_{j}}}^{\beta_{j}}+Q\left(\overline{B_{j}}, \eta_{j}\right) \sum_{j=1}^{N} \eta_{j}
$$

where again, $Q$ depends polynomially on $\overline{B_{j}}, \eta_{j}$, which implies $\left|Q\left(\bar{B}_{j}, \eta_{j}\right)\right| \leq$ $C_{1}\left(M_{0}\right)$ on $G$. Therefore,

$$
\begin{aligned}
\int_{G}\left(\prod_{i=1}^{M}{\overline{A_{i}}}^{\alpha_{i}}-B^{\beta}\right)^{2} \mathrm{~d} x & =\int_{G}\left(\prod_{i=1}^{M} \overline{A_{i}}{ }^{\alpha_{i}}-\prod_{j=1}^{N} \overline{B_{j}} \beta_{j}-Q\left(\overline{B_{j}}, \eta_{j}\right) \sum_{j=1}^{N} \eta_{j}\right)^{2} \mathrm{~d} x \\
& \geq \frac{1}{2} \int_{G}\left(\prod_{i=1}^{M} \overline{A_{i}}{ }^{\alpha_{i}}-\prod_{j=1}^{N}{\overline{B_{j}}}^{\beta_{j}}\right)^{2} \mathrm{~d} x
\end{aligned}
$$




$$
\begin{aligned}
& -\int_{G}\left|Q\left(\overline{B_{j}}, \eta_{j}\right)\right|^{2}\left|\sum_{j=1}^{N} \eta_{j}\right|^{2} \mathrm{~d} x \\
& \geq \frac{1}{2} \int_{G}\left(\prod_{i=1}^{M}{\overline{A_{i}}}^{\alpha_{i}}-\prod_{j=1}^{N}{\overline{B_{j}}}^{\beta_{j}}\right)^{2} \mathrm{~d} x-C_{1}\left(M_{0}\right)^{2} N^{2} \varepsilon
\end{aligned}
$$

where we used that $\left\|\eta_{j}\right\|^{2} \leq \varepsilon$ for all $j=1, \ldots, N$.

Combining these two estimates, we arrive at

$$
\begin{aligned}
\left\|A^{\alpha}-B^{\beta}\right\|^{2} \geq & \frac{1}{4}|G|\left(\prod_{i=1}^{M} \overline{A_{i}}{ }^{\alpha}-\prod_{j=1}^{N} \overline{B_{j}} \beta_{j}\right)^{2} \\
& -\varepsilon\left(\frac{1}{2} C_{1}\left(M_{0}\right)^{2} N^{2}+C_{0}\left(M_{0}\right)^{2} M^{2}\right) .
\end{aligned}
$$

By Jensen's inequality and the assumption of the Lemma, we have

$$
\overline{B_{j}} \leq \sqrt{\overline{B_{j}^{2}}} \leq \sqrt{\varepsilon}, \quad \forall j=1, \ldots, J
$$

On the other hand $\overline{B_{j}} \leq \sqrt{\overline{B_{j}^{2}}} \leq M_{0}, \forall j=J+1, \ldots, N$. Thus, the conservation law (50) and $\left\|\delta_{i}\right\|^{2} \leq \varepsilon$ yield

$$
\begin{aligned}
\bar{A}_{i} & =\sqrt{\overline{A_{i}^{2}}-\left\|\delta_{i}\right\|^{2}}=\sqrt{\frac{1}{\beta_{1}}\left(M_{i 1}-\alpha_{i} \overline{B_{1}^{2}}\right)-\left\|\delta_{i}\right\|^{2}} \\
& \geq \sqrt{\frac{M_{i 1}}{\beta_{1}}-\frac{\alpha_{i}}{\beta_{1}} \varepsilon-\varepsilon \quad \forall i=1, \ldots, M .}
\end{aligned}
$$

Hence, by using $|G| \geq \frac{1}{2}$, we get from (52) that

$\left\|A^{\alpha}-B^{\beta}\right\|^{2} \geq \frac{1}{8}\left[\prod_{i=1}^{M}\left(\frac{M_{i 1}}{\beta_{1}}-\frac{\alpha_{i}}{\beta_{1}} \varepsilon-\varepsilon\right)^{\alpha_{i} / 2}-\prod_{j=1}^{J}(\sqrt{\varepsilon})^{\beta_{j}} \prod_{j=J+1}^{N} M_{0}^{\beta_{j}}\right]^{2}-C_{2} \varepsilon$.

Because the right-hand side of the above inequality converges to $\frac{1}{8} \prod_{i=1}^{M}\left(\frac{M_{i 1}}{\beta_{1}}\right)^{\alpha_{i}}$ as $\varepsilon \rightarrow 0$, we can choose $\varepsilon>0$ small enough, but still explicit, such that

$$
\left\|A^{\alpha}-B^{\beta}\right\|^{2} \geq \frac{1}{16} \prod_{i=1}^{M}\left(\frac{M_{i 1}}{\beta_{1}}\right)^{\alpha_{i}} \geq \frac{1}{16 M_{0}^{2} J} \prod_{i=1}^{M}\left(\frac{M_{i 1}}{\beta_{1}}\right)^{\alpha_{i}} \sum_{j=1}^{J}\left\|\eta_{j}\right\|^{2}
$$

which implies the desired inequality (51) with the constant

$$
K_{1}=\frac{1}{16 M_{0}^{2} J} \prod_{i=1}^{M}\left(\frac{M_{i 1}}{\beta_{1}}\right)^{\alpha_{i}}
$$


Lemma 3.3. (A time-dependent entropy-entropy production estimate) Let $(a, b)=$ $\left(a_{1}, \ldots, a_{M}, b_{1}, \ldots, b_{N}\right)$ with $a_{i}, b_{j}: Q_{T} \rightarrow \mathbb{R}_{+}$be non-negative functions, which satisfy the conservation laws (3). Moreover,

$$
\left\|a_{i}\right\|_{L^{\infty}\left(Q_{T}\right)} \leq C_{T} \quad \text { and } \quad\left\|b_{j}\right\|_{L^{\infty}\left(Q_{T}\right)} \leq C_{T} \quad \text { for all } i, j .
$$

Then, there exists a constant $K_{2}>0$ independent of $T$ such that,

$$
D[a(T), b(T)] \geq K_{2} \frac{1}{1+\ln (1+T)}\left(E[a(T), b(T)]-E\left[a_{\infty}, b_{\infty}\right]\right) .
$$

Proof. Let $\varepsilon>0$ be a small constant chosen in Lemma 3.2. We will consider two cases and for convenience we will drop $T$ in $a_{i}(T)$ and $b_{j}(T)$ when there is no confusion. Case 1. Assume $\bar{a}_{i} \geq \varepsilon$ for all $i=1, \ldots, M$ and $\bar{b}_{j} \geq \varepsilon$ for all $j=1, \ldots, N$. By applying (48), we have

$$
\begin{aligned}
D[a, b] \geq & \sum_{i=1}^{M} d_{i} C\left(\Omega, m_{i}\right) \varepsilon^{m_{i}-1} \int_{\Omega} a_{i} \ln \frac{a_{i}}{\bar{a}_{i}} \mathrm{~d} x \\
& +\sum_{j=1}^{N} h_{j} C\left(\Omega, p_{j}\right) \varepsilon^{p_{j}-1} \int_{\Omega} b_{j} \ln \frac{b_{j}}{\bar{b}_{j}} \mathrm{~d} x+\int_{\Omega}\left(a^{\alpha}-b^{\beta}\right) \ln \frac{a^{\alpha}}{b^{\beta}} \mathrm{d} x \\
\geq & K_{3}\left[\sum_{i=1}^{M} \int_{\Omega} a_{i} \ln \frac{a_{i}}{\bar{a}_{i}} \mathrm{~d} x+\sum_{j=1}^{N} \int_{\Omega} b_{j} \ln \frac{b_{j}}{\bar{b}_{j}} \mathrm{~d} x+\int_{\Omega}\left(a^{\alpha}-b^{\beta}\right) \ln \frac{a^{\alpha}}{b^{\beta}} \mathrm{d} x\right]
\end{aligned}
$$

with

$$
K_{3}=\min _{i=1 \ldots M ; j=1 \ldots N}\left\{d_{i} C\left(\Omega, m_{i}\right) \varepsilon^{m_{i}-1} ; h_{j} C\left(\Omega, p_{j}\right) \varepsilon^{p_{j}-1} ; 1\right\}
$$

Using an entropy-entropy production inequality in case of system (R) with linear diffusion, see Lemma 4.1 below, we know that

$$
\begin{aligned}
& \sum_{i=1}^{M} \int_{\Omega} a_{i} \ln \frac{a_{i}}{\bar{a}_{i}} \mathrm{~d} x+\sum_{j=1}^{N} \int_{\Omega} b_{j} \ln \frac{b_{j}}{\bar{b}_{j}} \mathrm{~d} x+\int_{\Omega}\left(a^{\alpha}-b^{\beta}\right) \ln \frac{a^{\alpha}}{b^{\beta}} \mathrm{d} x \\
& \quad \geq K_{4}\left(E[a, b]-E\left[a_{\infty}, b_{\infty}\right]\right)
\end{aligned}
$$

for an explicit constant $K_{4}>0$. Therefore,

$$
D[a, b] \geq K_{3} K_{4}\left(E[a, b]-E\left[a_{\infty}, b_{\infty}\right]\right) .
$$

Case 2. Suppose either $\bar{a}_{i} \leq \varepsilon$ for some $i \in\{1, \ldots, M\}$ or $\bar{b}_{j} \leq \varepsilon$ for some $j=$ $1, \ldots, N$.

Due to the mass conservation laws $\beta_{j} \bar{a}_{i}+\alpha_{i} \bar{b}_{j}=M_{i j}$, it cannot happen that $\bar{a}_{i} \leq \varepsilon$ and $\bar{b}_{j} \leq \varepsilon$ simultaneously for a sufficiently small $\varepsilon$, e.g. $\varepsilon<\frac{M_{i j}}{2} \min \left\{\frac{1}{\beta_{j}} ; \frac{1}{\alpha_{i}}\right\}$. Therefore, without loss of generality, we can assume that

$$
\bar{b}_{j} \leq \varepsilon \quad \forall j=1, \ldots, J \quad \text { and } \quad \bar{b}_{j} \geq \varepsilon \quad \forall j=J+1, \ldots, N
$$


for some $J \in\{1, \ldots, N\}$. Moreover, by mass conservation laws

$$
\bar{a}_{i}=\frac{1}{\beta_{1}}\left(M_{i 1}-\alpha_{i} \bar{b}_{1}\right) \geq \frac{1}{\beta_{1}}\left(M_{i 1}-\alpha_{i} \varepsilon\right), \quad \text { for all } i=1, \ldots, M .
$$

Thus, we can apply Lemma 3.1 to $D[a, b]$ and estimate

$$
\begin{aligned}
D[a, b] \geq & \sum_{i=1}^{M} d_{i} C\left(\Omega, m_{i}\right)\left[\frac{1}{\beta_{1}}\left(M_{i 1}-\alpha_{i} \varepsilon\right)\right]^{m_{i}-1} \int_{\Omega} a_{i} \ln \frac{a_{i}}{\bar{a}_{i}} \mathrm{~d} x \\
& +\sum_{j=J+1}^{N} h_{j} C\left(\Omega, p_{j}\right) \varepsilon^{p_{j}-1} \int_{\Omega} b_{j} \ln \frac{b_{j}}{\bar{b}_{j}} \mathrm{~d} x+\int_{\Omega}\left(a^{\alpha}-b^{\beta}\right) \ln \frac{a^{\alpha}}{b^{\beta}} \mathrm{d} x \\
\geq & K_{5}\left[\sum_{i=1}^{M}\left\|\sqrt{a_{i}}-\overline{\sqrt{a_{i}}}\right\|^{2}+\sum_{j=J+1}^{N}\left\|\sqrt{b_{j}}-\overline{\sqrt{b_{j}}}\right\|^{2}+\left\|A^{\alpha}-B^{\beta}\right\|^{2}\right] \\
= & K_{5}\left[\sum_{i=1}^{M}\left\|\delta_{i}\right\|^{2}+\sum_{j=J+1}^{N}\left\|\eta_{j}\right\|^{2}+\left\|A^{\alpha}-B^{\beta}\right\|^{2}\right],
\end{aligned}
$$

where we have used $(x-y) \ln (x / y) \geq 4(\sqrt{x}-\sqrt{y})^{2}$ and $K_{5}=\min _{i=1 \ldots M ; j=J+1 \ldots N}\left\{d_{i} C\left(\Omega, m_{i}\right)\left[\frac{1}{\beta_{1}}\left(M_{i 1}-\alpha_{i} \varepsilon\right)\right]^{m_{i}-1} ; h_{j} C\left(\Omega, p_{j}\right) \varepsilon^{p_{j}-1} ; 4\right\}$.

Applying Lemma 3.2 yields

$$
D[a, b] \geq K_{6}\left[\sum_{i=1}^{M}\left\|\delta_{i}\right\|^{2}+\sum_{j=1}^{N}\left\|\eta_{j}\right\|^{2}+\left\|A^{\alpha}-B^{\beta}\right\|^{2}\right]
$$

where

$$
K_{6}=\frac{1}{2} \min \left\{K_{5} ; K_{5} K_{1}\right\}
$$

By using another functional inequality, which was already proven in the case of linear diffusion, see (61) in Sect. 4, we have

$$
\begin{aligned}
D[a, b] \geq & K_{7}\left[\sum_{i=1}^{M}\left(\left\|\delta_{i}\right\|^{2}+\left|\sqrt{\overline{A_{i}^{2}}}-A_{i, \infty}\right|^{2}\right)\right. \\
& \left.+\sum_{j=1}^{N}\left(\left\|\eta_{j}\right\|^{2}+\left|\sqrt{\overline{B_{j}^{2}}}-B_{j, \infty}\right|^{2}\right)\right] .
\end{aligned}
$$

Now, we estimate $E[a, b]-E\left[a_{\infty}, b_{\infty}\right]$ from above. Consider the two variables function

$$
\Phi(x, y)=\frac{x \ln (x / y)-x+y}{(\sqrt{x}-\sqrt{y})^{2}}
$$


which is continuous in $(0, \infty)^{2}$ and $\Phi(\cdot, y)$ is increasing for each fixed $y>0$. It holds that

$$
\begin{aligned}
E[a, b]-E\left[a_{\infty}, b_{\infty}\right] & \\
= & \sum_{i=1}^{M} \int_{\Omega} \Phi\left(a_{i}, a_{i, \infty}\right)\left(A_{i}-A_{i, \infty}\right)^{2} \mathrm{~d} x+\sum_{j=1}^{N} \int_{\Omega} \Phi\left(b_{j}, b_{j, \infty}\right)\left(B_{j}-B_{j, \infty}\right)^{2} \mathrm{~d} x \\
\leq & \max _{i=1 \ldots M ; j=1 \ldots N}\left\{\Phi\left(\left\|a_{i}\right\|_{L^{\infty}\left(Q_{T}\right)}, a_{i, \infty}\right) ; \Phi\left(\left\|b_{j}\right\|_{\left.\left.L^{\infty}\left(Q_{T}\right), b_{j, \infty}\right)\right\}}\right.\right. \\
& {\left[\sum_{i=1}^{M}\left\|A_{i}-A_{i, \infty}\right\|^{2}+\sum_{j=1}^{N}\left\|B_{j}-B_{j, \infty}\right\|^{2}\right] } \\
\leq & K_{8}(1+\ln (1+T))\left[\sum_{i=1}^{M}\left(\left\|\delta_{i}\right\|^{2}+\left|\bar{A}_{i}-A_{i, \infty}\right|^{2}\right)\right. \\
& \left.+\sum_{j=1}^{N}\left(\left\|\eta_{j}\right\|^{2}+\left|\bar{B}_{j}-B_{j, \infty}\right|^{2}\right)\right]
\end{aligned}
$$

where in the last inequality, we have used the estimates $\left\|a_{i}\right\|_{L^{\infty}\left(Q_{T}\right)} \leq C_{T}$ and $\left\|b_{j}\right\|_{L^{\infty}\left(Q_{T}\right)} \leq C_{T}$ and that $C_{T}$ is a constant growing at most polynomially w.r.t. $T$.

Next, from $\left\|\delta_{i}\right\|^{2}=\overline{A_{i}^{2}}-\bar{A}_{i}^{2}=\left(\sqrt{\overline{A_{i}^{2}}}-\bar{A}_{i}\right)\left(\sqrt{\overline{A_{i}^{2}}}+\bar{A}_{i}\right)$, we have

$$
\bar{A}_{i}=\sqrt{\overline{\overline{A_{i}^{2}}}}-\frac{\left\|\delta_{i}\right\|^{2}}{\sqrt{\overline{A_{i}^{2}}}+\bar{A}_{i}}=\sqrt{\overline{A_{i}^{2}}}-Q_{i}\left(A_{i}\right)\left\|\delta_{i}\right\| \quad \text { with } \quad Q_{i}\left(A_{i}\right)=\frac{\left\|\delta_{i}\right\|}{\sqrt{\overline{A_{i}^{2}}}+\bar{A}_{i}} .
$$

It's obvious that $Q\left(A_{i}\right) \geq 0$ and moreover

$$
Q_{i}\left(A_{i}\right)^{2}=\frac{\overline{A_{i}^{2}}-\bar{A}_{i}^{2}}{\left(\sqrt{\overline{A_{i}^{2}}}+\bar{A}_{i}\right)^{2}}=\frac{\sqrt{\overline{A_{i}^{2}}}-\bar{A}_{i}}{\sqrt{\overline{A_{i}^{2}}}+\bar{A}_{i}} \leq 1 .
$$

Therefore,

$$
\begin{aligned}
\left|\bar{A}_{i}-A_{i, \infty}\right|^{2} & \leq 2\left(\left|\sqrt{\overline{A_{i}^{2}}}-\bar{A}_{i}\right|^{2}+\left|\sqrt{\overline{A_{i}^{2}}}-A_{i, \infty}\right|^{2}\right) \\
& =2\left(Q_{i}\left(A_{i}\right)^{2}\left\|\delta_{i}\right\|^{2}+\left|\sqrt{\overline{A_{i}^{2}}}-A_{i, \infty}\right|^{2}\right) \\
& \leq 2\left(\left\|\delta_{i}\right\|^{2}+\left|\sqrt{\overline{A_{i}^{2}}}-A_{i, \infty}\right|^{2}\right) \quad \text { for all } i=1 \ldots M
\end{aligned}
$$

and similarly

$$
\left|\bar{B}_{j}-B_{j, \infty}\right|^{2} \leq 2\left(\left\|\eta_{i}\right\|^{2}+\left|\sqrt{\overline{B_{j}^{2}}}-B_{j, \infty}\right|^{2}\right) \quad \text { for all } j=1 \ldots N .
$$


Hence, it follows from (54) that

$$
\begin{aligned}
E[a, b]-E\left[a_{\infty}, b_{\infty}\right] \leq & 3 K_{8}(1+\ln (1+T))\left[\sum_{i=1}^{M}\left(\left\|\delta_{i}\right\|^{2}+\left|\sqrt{\overline{A_{i}^{2}}}-A_{i, \infty}\right|^{2}\right)\right. \\
& \left.+\sum_{j=1}^{N}\left(\left\|\eta_{j}\right\|^{2}+\left|\sqrt{\overline{B_{j}^{2}}}-B_{j, \infty}\right|^{2}\right)\right] .
\end{aligned}
$$

A combination of (53) and (55) yields

$$
D[a, b] \geq \frac{K_{7}}{3 K_{8}(1+\ln (1+T))}\left(E[a, b]-E\left[a_{\infty}, b_{\infty}\right]\right) .
$$

Finally, from Case 1 and Case 2, we can conclude the proof of Lemma 3.3 with

$$
K_{2}=\min \left\{K_{3} K_{4} ; \frac{K_{7}}{3 K_{8}}\right\} \text {. }
$$

Remark 3.2. The assumptions $\left\|a_{i}\right\|_{L^{\infty}\left(Q_{T}\right)} \leq C_{T}$ and $\left\|b_{j}\right\|_{L^{\infty}\left(Q_{T}\right)} \leq C_{T}$ in Lemma 3.3 are only needed to estimate $E[a, b]-E\left[a_{\infty}, b_{\infty}\right]$ above as in (54). In the case of linear diffusion, it is possible to avoid these $L^{\infty}$-bounds by using the additivity of the relative entropy (see also the proof of Lemma 4.1 in Sect. 4), i.e.

$$
E[a, b]-E\left[a_{\infty}, b_{\infty}\right]=(E[a, b]-E[\bar{a}, \bar{b}])+\left(E[\bar{a}, \bar{b}]-E\left[a_{\infty}, b_{\infty}\right]\right) .
$$

However, while for linear diffusion, the logarithmic Sobolev inequality controls to first part $E[a, b]-E[\bar{a}, \bar{b}] \leq C\left(C_{\mathrm{LSI}}\right) D[a, b]$, such an estimate is unclear in the case of porous media diffusion, where the generalised logarithmic Sobolev inequality in Lemma 3.1 degenerates for states without lower bounds on the spatial averages.

We need also the following Csiszár-Kullback-Pinsker type inequality. The proof is standard and can be found in e.g. [13,19].

Lemma 3.4. There exists a constant $C_{\mathrm{CKP}}>0$ such that for any measurable nonnegative functions $a_{i}, b_{j}: \Omega \rightarrow \mathbb{R}_{+}$satisfying the mass conservation (49), there holds

$$
E[a, b]-E\left[a_{\infty}, b_{\infty}\right] \geq C_{\mathrm{CKP}}\left(\sum_{i=1}^{M}\left\|a_{i}-a_{i, \infty}\right\|_{1}^{2}+\sum_{j=1}^{N}\left\|b_{j}-b_{j, \infty}\right\|_{1}^{2}\right) .
$$

We are ready to prove Theorem 1.3.

Proof of Theorem 1.3. Due to the condition

$$
m_{i}, p_{j}>\max \left\{v-\min \left\{\frac{4}{d+2} ; 1\right\} ; 1\right\} \quad \forall i=1 \ldots M, j=1 \ldots N,
$$


we can apply Theorem 1.2 to show boundedness of the weak solution $(a, b)$ to (R), i.e.

$$
\left\|a_{i}\right\|_{L^{\infty}\left(Q_{T}\right)} \leq C_{T}, \quad\left\|b_{j}\right\|_{L^{\infty}\left(Q_{T}\right)} \leq C_{T}, \quad \forall i=1 \ldots M, j=1 \ldots N
$$

By applying Lemma 3.3, this yields

$$
D[a(T), b(T)] \geq K_{2} \frac{1}{1+\ln (1+T)}\left(E[a(T), b(T)]-E\left[a_{\infty}, b_{\infty}\right]\right) .
$$

Moreover, due to the boundedness of solutions, we have the entropy-entropy production relation

$$
\begin{aligned}
\frac{\mathrm{d}}{\mathrm{d} t}\left(E[a, b]-E\left[a_{\infty}, b_{\infty}\right]\right) & =\frac{\mathrm{d}}{\mathrm{d} t} E[a, b]=-D[a, b] \\
& \leq-K_{2} \frac{1}{1+\ln (1+T)}\left(E[a, b]-E\left[a_{\infty}, b_{\infty}\right]\right) .
\end{aligned}
$$

A classical Gronwall's inequality leads to

$$
\begin{aligned}
& E[a(T), b(T)]-E\left[a_{\infty}, b_{\infty}\right] \\
& \quad \leq \exp \left(-K_{2} \int_{0}^{T} \frac{d \tau}{1+\ln (1+\tau)}\right)\left(E\left[a_{0}, b_{0}\right]-E\left[a_{\infty}, b_{\infty}\right]\right) .
\end{aligned}
$$

By direct calculations,

$$
\exp \left(-K_{2} \int_{0}^{T} \frac{d \tau}{1+\ln (1+\tau)}\right) \geq \exp \left(-K_{2} \int_{0}^{T} \frac{d \tau}{1+\tau}\right)=(1+T)^{-K_{2}} .
$$

Hence,

$$
E[a(T), b(T)]-E\left[a_{\infty}, b_{\infty}\right] \leq(1+T)^{-K_{2}}\left(E\left[a_{0}, b_{0}\right]-E\left[a_{\infty}, b_{\infty}\right]\right),
$$

and therefore thanks to the Csiszár-Kullback-Pinsker inequality in Lemma 3.4

$$
\begin{aligned}
& \sum_{i=1}^{M}\left\|a_{i}(T)-a_{i, \infty}\right\|_{1}^{2}+\sum_{j=1}^{N}\left\|b_{j}(T)-b_{j, \infty}\right\|_{1}^{2} \\
& \quad \leq C_{\mathrm{CKP}}^{-1}(1+T)^{-K_{2}}\left(E\left[a_{0}, b_{0}\right]-E\left[a_{\infty}, b_{\infty}\right]\right)
\end{aligned}
$$

which implies algebraic convergence to equilibrium of solutions to $(\mathrm{R})$.

We will now show that from this it is possible to recover exponential convergence. Since the right-hand side of (57) tends to zero as $T \rightarrow \infty$, we can choose

$$
T_{0}=\max \left\{1 ;\left[\frac{C_{\mathrm{CKP}}^{-1}\left(E\left[a_{0}, b_{0}\right]-E\left[a_{\infty}, b_{\infty}\right]\right)}{\frac{1}{2} \min _{i=1 \ldots M ; j=1 \ldots N}\left\{a_{i, \infty}^{2}, b_{j, \infty}^{2}\right\}}\right]^{1 / K_{2}}-1\right\}
$$

which implies for all $t \geq T_{0}$

$$
\left\|a_{i}(t)-a_{i, \infty}\right\|_{1} \leq \frac{1}{2} a_{i, \infty} \quad \text { and } \quad\left\|b_{j}(t)-b_{j, \infty}\right\|_{1} \leq \frac{1}{2} b_{j, \infty},
$$


and thus,

$$
\bar{a}_{i}(t)=\left\|a_{i}(t)\right\|_{1} \geq \frac{1}{2} a_{i, \infty} \quad \text { and } \quad \bar{b}_{j}(t)=\left\|b_{j}(t)\right\|_{1} \geq \frac{1}{2} b_{j, \infty} \quad \text { for all } \quad t \geq T_{0} .
$$

Therefore, for all $t \geq T_{0}$, we can apply these lower bounds on the spatial averages bounds and Lemma 3.1 to estimate the entropy-entropy production as follows

$$
\begin{aligned}
D[a(t), b(t)] \geq & C_{1}\left[\sum_{i=1}^{M} \int_{\Omega} a_{i} \ln \frac{a_{i}}{\overline{a_{i}}} \mathrm{~d} x+\sum_{j=1}^{N} \int_{\Omega} b_{j} \ln \frac{b_{j}}{\overline{b_{j}}} \mathrm{~d} x\right. \\
& \left.+\int_{\Omega}\left(a^{\alpha}-b^{\beta}\right) \ln \frac{a^{\alpha}}{b^{\beta}} \mathrm{d} x\right] \text { for all } t \geq T_{0},
\end{aligned}
$$

with

$C_{1}=\min _{i=1 \ldots M ; j=1 \ldots N}\left\{d_{i} C\left(\Omega, m_{i}\right)\left(\frac{1}{2} a_{i, \infty}\right)^{m_{i}-1} ; h_{j} C\left(\Omega, p_{j}\right)\left(\frac{1}{2} b_{j, \infty}\right)^{p_{j}-1} ; 1\right\}$.

By applying again Lemma 4.1, we obtain

$$
D[a(t), b(t)] \geq C_{1} \lambda\left(E[a(t), b(t)]-E\left[a_{\infty}, b_{\infty}\right]\right) \quad \text { for all } \quad t \geq T_{0},
$$

which in a combination with the classical Gronwall's inequality yields for all $t \geq T_{0}$,

$$
\begin{aligned}
E[a(t), b(t)]-E\left[a_{\infty}, b_{\infty}\right] & \leq e^{-\lambda C_{1}\left(t-T_{0}\right)}\left(E\left[a\left(T_{0}\right), b\left(T_{0}\right)\right]-E\left[a_{\infty}, b_{\infty}\right]\right) \\
& \leq e^{-\lambda C_{1} t} e^{\lambda C_{1} T_{0}}\left(1+T_{0}\right)^{-K_{2}}\left(E\left[a_{0}, b_{0}\right]-E\left[a_{\infty}, b_{\infty}\right]\right) \\
& \leq e^{-\lambda C_{1} t} e^{\lambda C_{1} T_{0}}\left(E\left[a_{0}, b_{0}\right]-E\left[a_{\infty}, b_{\infty}\right]\right)
\end{aligned}
$$

where we used (56) for the second inequality. On the other hand, it follows from (56) that for all $0 \leq t<T_{0}$,

$$
\begin{aligned}
E[a(t), b(t)]-E\left[a_{\infty}, b_{\infty}\right] & \leq(1+t)^{-K_{2}}\left(E\left[a_{0}, b_{0}\right]-E\left[a_{\infty}, b_{\infty}\right]\right) \\
& \leq e^{-\lambda C_{1} t} e^{\lambda C_{1} T_{0}}\left(E\left[a_{0}, b_{0}\right]-E\left[a_{\infty}, b_{\infty}\right]\right)
\end{aligned}
$$

Due to the explicitness of $T_{0}$ in (58), we eventually get the exponential convergence

$$
E[a(t), b(t)]-E\left[a_{\infty}, b_{\infty}\right] \leq C_{2} e^{-\widehat{\lambda} t}\left(E\left[a_{0}, b_{0}\right]-E\left[a_{\infty}, b_{\infty}\right]\right) \quad \text { for all } t \geq 0
$$

with the constant $C_{2}=e^{\lambda C_{1} T_{0}}$ and the rate $\widehat{\lambda}=\lambda C_{1}$. Note that $C_{2}$ is explicit since $T_{0}$ is explicit (see (58)). With another application of the Csiszár-Kullback-Pinsker inequality in Lemma 3.4, this yields 


$$
\begin{aligned}
& \sum_{i=1}^{M}\left\|a_{i}(t)-a_{i, \infty}\right\|_{1}^{2}+\sum_{j=1}^{N}\left\|b_{j}(t)-b_{j, \infty}\right\|_{1}^{2} \\
& \quad \leq C_{2} C_{\mathrm{CKP}}^{-1} e^{-\widehat{\lambda} t}\left(E\left[a_{0}, b_{0}\right]-E\left[a_{\infty}, b_{\infty}\right]\right) \leq C_{3} e^{-\widehat{\lambda} t}
\end{aligned}
$$

with $C_{3}=C_{2} C_{\mathrm{CKP}}^{-1}\left(E\left[a_{0}, b_{0}\right]-E\left[a_{\infty}, b_{\infty}\right]\right)$. Finally, by combining the above exponential $L^{1}$-convergence with the at most polynomial grow $L^{\infty}$ a priori estimates $\left\|a_{i}\right\|_{L^{\infty}\left(Q_{T}\right)},\left\|b_{j}\right\|_{L^{\infty}\left(Q_{T}\right)} \leq C_{T}$, interpolation yields for any $1<p<\infty$,

$$
\begin{aligned}
\left\|a_{i}(T)-a_{i, \infty}\right\|_{p} & \leq\left\|a_{i}(T)-a_{i, \infty}\right\|_{\infty}^{\theta}\left\|a_{i}(T)-a_{i, \infty}\right\|_{1}^{1-\theta} \\
& \leq C_{T}^{\theta} C_{3}^{1-\theta} e^{-\widehat{\lambda}(1-\theta) T} \leq C_{4} e^{-\lambda_{p} T}
\end{aligned}
$$

for some $0<\lambda_{p}<\widehat{\lambda}(1-\theta)$ since $C_{T}$ grows at most polynomially in $T$, and similarly

$$
\left\|b_{j}(T)-b_{j, \infty}\right\|_{p} \leq\left\|b_{j}(T)-b_{j, \infty}\right\|_{\infty}^{\theta}\left\|b_{j}(T)-b_{j, \infty}\right\|_{1}^{1-\theta} \leq C_{5} e^{-\lambda_{p} T} .
$$

This concludes the proof of Theorem 1.3.

\section{Entropy-entropy production inequality}

Lemma 4.1. (Entropy-entropy production estimate) Let $a_{\infty} \in(0, \infty)^{M}$ and $b_{\infty} \in$ $(0, \infty)^{N}$ satisfy

$$
a_{\infty}^{\alpha}=b_{\infty}^{\beta}
$$

where $\alpha \in[1, \infty)^{M}$ and $\beta \in[1, \infty)^{N}$.

Then, there exists an explicit constant $\lambda>0$ depending on $a_{\infty}, b_{\infty}, \alpha, \beta$ and the domain $\Omega$, such that for any non-negative functions $a=\left(a_{i}\right): \Omega \rightarrow \mathbb{R}_{+}^{M}$ and $b=\left(b_{j}\right): \Omega \rightarrow \mathbb{R}_{+}^{N}$ satisfying

$$
\beta_{j} \bar{a}_{i}+\alpha_{i} \bar{b}_{j}=\beta_{j} a_{i, \infty}+\alpha_{i} b_{j, \infty} \quad \text { for all } i=1, \ldots, M, j=1, \ldots, N,
$$

the following entropy-entropy production inequality holds

$$
\widetilde{D}[a, b] \geq \lambda\left(E[a, b]-E\left[a_{\infty}, b_{\infty}\right]\right)
$$

where

$$
\widetilde{D}[a, b]=\sum_{i=1}^{M} \int_{\Omega} a_{i} \ln \frac{a_{i}}{\bar{a}_{i}} d x+\sum_{j=1}^{N} \int_{\Omega} b_{j} \ln \frac{b_{j}}{\bar{b}_{j}} d x+\int_{\Omega}\left(a^{\alpha}-b^{\beta}\right) \ln \frac{a^{\alpha}}{b^{\beta}} d x
$$

and

$$
E[a, b]=\sum_{i=1}^{M} \int_{\Omega}\left(a_{i} \ln a_{i}-a_{i}+1\right) d x+\sum_{j=1}^{N} \int_{\Omega}\left(b_{j} \ln b_{j}-b_{j}+1\right) d x .
$$


Remark 4.1. The above entropy-entropy production inequality was first proved in [19] in a constructive way with explicit bounds on the constant $\lambda$. The proof stated here follows the line of a significantly simplified version presented in [20].

Proof. First, by the additivity of the relative entropy, we have

$$
\begin{aligned}
E[a, b]-E\left[a_{\infty}, b_{\infty}\right]= & (E[a, b]-E[\bar{a}, \bar{b}])+\left(E[\bar{a}, \bar{b}]-E\left[a_{\infty}, b_{\infty}\right]\right) \\
= & {\left[\sum_{i=1}^{M} \int_{\Omega} a_{i} \ln \frac{a_{i}}{\bar{a}_{i}} \mathrm{~d} x+\sum_{j=1}^{N} \int_{\Omega} b_{j} \ln \frac{b_{j}}{\bar{b}_{j}} \mathrm{~d} x\right] } \\
& +\left[\sum_{i=1}^{M}\left(\bar{a}_{i} \ln \frac{\bar{a}_{i}}{a_{i, \infty}}-\bar{a}_{i}+a_{i, \infty}\right)\right. \\
& \left.+\sum_{j=1}^{N}\left(\bar{b}_{j} \ln \frac{\bar{b}_{j}}{b_{j, \infty}}-\bar{b}_{j}+b_{j, \infty}\right)\right] \\
= & :(I)+(I I) .
\end{aligned}
$$

It is straightforward that $(I)$ can be controlled by $\widetilde{D}[a, b]$, i.e.

$$
\frac{1}{2} \widetilde{D}[a, b] \geq \frac{1}{2} \times(I) .
$$

It remains to control $(I I)$. To do that, we first introduce the following useful notations and definitions

$$
\begin{aligned}
A_{i} & =\sqrt{a_{i}}, \quad B_{j}=\sqrt{b_{j}}, \quad A_{i, \infty}=\sqrt{a_{i, \infty}}, \quad B_{j, \infty}=\sqrt{b_{j, \infty}}, \\
\delta_{i}(x) & =A_{i}(x)-\bar{A}_{i}, \quad \eta_{j}(x)=B_{j}(x)-\bar{B}_{j},
\end{aligned}
$$

and

$$
A^{\alpha}=\prod_{i=1}^{M} A_{i}^{\alpha_{i}}, \quad B^{\beta}=\prod_{j=1}^{N} B_{j}^{\beta_{j}}
$$

By the elementary inequality $(x-y) \ln (x / y) \geq 4(\sqrt{x}-\sqrt{y})^{2}$, we have

$$
\int_{\Omega} a_{i} \ln \frac{a_{i}}{\bar{a}_{i}} \mathrm{~d} x=\int_{\Omega}\left(a_{i} \ln \frac{a_{i}}{\bar{a}_{i}}-a_{i}+\bar{a}_{i}\right) \mathrm{d} x \geq 4 \int_{\Omega}\left(\sqrt{a_{i}}-\sqrt{\bar{a}_{i}}\right)^{2} \mathrm{~d} x \geq 4\left\|\delta_{i}\right\|^{2}
$$

and similarly $\int_{\Omega} b_{j} \ln \frac{b_{j}}{\bar{b}_{j}} \mathrm{~d} x \geq 4\left\|\eta_{j}\right\|^{2}$. Moreover, $\int_{\Omega}\left(a^{\alpha}-b^{\beta}\right) \ln \frac{a^{\alpha}}{b^{\beta}} \mathrm{d} x \geq 4 \| A^{\alpha}-$ $B^{\beta} \|^{2}$. Therefore,

$$
\frac{1}{2} \widetilde{D}[a, b] \geq 2\left[\sum_{i=1}^{M}\left\|\delta_{i}\right\|^{2}+\sum_{j=1}^{N}\left\|\eta_{j}\right\|^{2}+\left\|A^{\alpha}-B^{\beta}\right\|^{2}\right] .
$$


In order to bound to estimate the right-hand side of (59) with an upper bound of (II), we first observe from the conservation laws

$$
\beta_{j} \bar{a}_{i}+\alpha_{i} \bar{b}_{j}=\beta_{j} a_{i, \infty}+\alpha_{i} b_{j, \infty}, \quad \text { for all } i, j .
$$

that there exists a constant $M_{0}>0$ such that

$$
\bar{a}_{i}, \bar{b}_{j} \leq M_{0}^{2}, \quad \text { for all } i, j .
$$

Next, we note that the two variables function

$$
\Phi(x, y)=\frac{x \ln (x / y)-x+y}{(\sqrt{x}-\sqrt{y})^{2}}
$$

is continuous on $(0, \infty)^{2}$, and $\Phi(\cdot, y)$ is increasing for each fixed $y$. Then, the term $(I I)$ is estimated as

$$
\begin{aligned}
(I I)= & \sum_{i=1}^{M} \Phi\left(\bar{a}_{i}, a_{i, \infty}\right)\left(\sqrt{\bar{a}_{i}}-\sqrt{a_{i, \infty}}\right)^{2}+\sum_{j=1}^{N} \Phi\left(\bar{b}_{j}, b_{j, \infty}\right)\left(\sqrt{\bar{b}_{j}}-\sqrt{b_{j, \infty}}\right)^{2} \\
\leq & \max _{i, j}\left\{\Phi\left(M_{0}^{2}, a_{i, \infty}\right) ; \Phi\left(M_{0}^{2}, b_{j, \infty}\right)\right\}\left(\sum_{i=1}^{M}\left(\sqrt{\overline{A_{i}^{2}}}-A_{i, \infty}\right)^{2}\right. \\
& \left.+\sum_{j=1}^{N}\left(\sqrt{\overline{B_{j}^{2}}}-B_{j, \infty}\right)^{2}\right) .
\end{aligned}
$$

From (59) and (60), it remains to show that

$$
\begin{aligned}
& \sum_{i=1}^{M}\left\|\delta_{i}\right\|^{2}+\sum_{j=1}^{N}\left\|\eta_{j}\right\|^{2}+\left\|A^{\alpha}-B^{\beta}\right\|^{2} \\
& \geq C_{0}\left(\sum_{i=1}^{M}\left(\sqrt{\overline{A_{i}^{2}}}-A_{i, \infty}\right)^{2}+\sum_{j=1}^{N}\left(\sqrt{\overline{B_{j}^{2}}}-B_{j, \infty}\right)^{2}\right)
\end{aligned}
$$

for some constant $C_{0}>0$. By using Lemma 4.2, we have with $\bar{A}=\left(\bar{A}_{1}, \ldots, \bar{A}_{M}\right)$ and $\bar{B}=\left(\bar{B}_{1}, \ldots, \bar{B}_{N}\right)$

$$
\begin{aligned}
& \sum_{i=1}^{M}\left\|\delta_{i}\right\|^{2}+\sum_{j=1}^{N}\left\|\eta_{j}\right\|^{2}+\left\|A^{\alpha}-B^{\beta}\right\|^{2} \\
& \geq C_{1}\left(\sum_{i=1}^{M}\left\|\delta_{i}\right\|^{2}+\sum_{j=1}^{N}\left\|\eta_{j}\right\|^{2}+\left|\bar{A}^{\alpha}-\bar{B}^{\beta}\right|^{2}\right)
\end{aligned}
$$

for some constant $C_{1}>0$. Using the ansatz $\overline{A_{i}^{2}}=A_{i, \infty}^{2}\left(1+\mu_{i}\right)^{2}$ and $\overline{B_{j}^{2}}=B_{j, \infty}^{2}\left(1+\zeta_{j}\right)^{2}, \quad$ where $\mu_{i}, \zeta_{j} \in[-1, \infty)$, 
the right-hand side of (61) writes as

$$
\text { RHS of }(61)=C_{0}\left(\sum_{i=1}^{M} \mu_{i}^{2}+\sum_{j=1}^{N} \zeta_{j}^{2}\right)
$$

Moreover, the bounds $\overline{a_{i}}=\overline{A_{i}^{2}} \leq M_{0}^{2}$ and $\overline{b_{j}}=\overline{B_{j}^{2}} \leq M_{0}^{2}$ imply

$$
-1 \leq \mu_{i} \leq M_{1} \text { and }-1 \leq \zeta_{j} \leq M_{1}
$$

for some constant $M_{1}>0$. From the ansatz (63) (and similar to the proof of Lemma 3.3), we have

$$
\begin{aligned}
& \bar{A}_{i}=\sqrt{\overline{\overline{A_{i}^{2}}}}-Q_{i}\left(A_{i}\right)\left\|\delta_{i}\right\|=A_{i, \infty}\left(1+\mu_{i}\right)-Q_{i}\left(A_{i}\right)\left\|\delta_{i}\right\| \\
& \overline{B_{j}}=\sqrt{\overline{B_{j}^{2}}}-R_{j}\left(B_{j}\right)\left\|\eta_{j}\right\|=B_{j, \infty}\left(1+\zeta_{j}\right)-R_{j}\left(B_{j}\right)\left\|\eta_{j}\right\|
\end{aligned}
$$

where

$$
0 \leq Q_{i}\left(A_{i}\right):=\frac{\left\|\delta_{i}\right\|}{\sqrt{\overline{A_{i}^{2}}}+\bar{A}_{i}} \leq 1 \quad \text { and } \quad 0 \leq R_{j}\left(B_{j}\right):=\frac{\left\|\eta_{j}\right\|}{\sqrt{\overline{B_{j}^{2}}}+\bar{B}_{j}} \leq 1 .
$$

Next, we use Taylor expansion to estimate

$$
{\overline{A_{i}}}^{\alpha_{i}}=\left(A_{i, \infty}\left(1+\mu_{i}\right)-Q_{i}\left(A_{i}\right)\left\|\delta_{i}\right\|\right)^{\alpha_{i}}=A_{i, \infty}^{\alpha_{i}}\left(1+\mu_{i}\right)^{\alpha_{i}}+\widehat{Q}_{i}\left\|\delta_{i}\right\|
$$

in which the Lagrange remainder term $\widehat{Q}_{i}=\widehat{Q}\left(\mu_{i},\left\|\delta_{i}\right\|\right)$ is uniformly bounded above by a constant for all admissible values of $\mu_{i}$ and $\left\|\delta_{i}\right\|$ thanks to the boundedness of $\mu_{i}$ and $\left\|\delta_{i}\right\| \leq \sqrt{\overline{A_{i}^{2}}} \leq M_{0}$. Similarly,

$$
{\overline{B_{j}}}^{\beta_{j}}=B_{j, \infty}^{\beta_{j}}\left(1+\zeta_{j}\right)^{\beta_{j}}+\widehat{R}_{j}\left\|\eta_{j}\right\|
$$

with uniformly bounded remainder $\widehat{R}_{j}\left(\zeta_{j},\left\|\eta_{j}\right\|\right)$. Thus,

$$
\begin{aligned}
\left|\bar{A}^{\alpha}-\bar{B}^{\beta}\right|^{2}= & \left|\prod_{i=1}^{M} \bar{A}_{i}^{\alpha_{i}}-\prod_{j=1}^{N} \bar{B}_{j}^{\beta_{j}}\right|^{2} \\
= & \mid \prod_{i=1}^{M}\left(A_{i, \infty}^{\alpha_{i}}\left(1+\mu_{i}\right)^{\alpha_{i}}+\widehat{Q}_{i}\left\|\delta_{i}\right\|\right) \\
& -\left.\prod_{j=1}^{N}\left(B_{j, \infty}^{\beta_{j}}\left(1+\zeta_{j}\right)^{\beta_{j}}+\widehat{R}_{j}\left\|\eta_{j}\right\|\right)\right|^{2} \\
= & \mid A_{\infty}^{\alpha} \prod_{i=1}^{M}\left(1+\mu_{i}\right)^{\alpha_{i}}-B_{\infty}^{\beta} \prod_{j=1}^{N}\left(1+\zeta_{j}\right)^{\beta_{j}} \\
& +\left.\Theta\left(\widehat{Q}_{i}, \widehat{R}_{j}\right)\left(\sum_{i=1}^{M}\left\|\delta_{i}\right\|+\sum_{j=1}^{N}\left\|\eta_{j}\right\|\right)\right|^{2}
\end{aligned}
$$


with $\Theta\left(\widehat{Q}_{i}, \widehat{R}_{j}\right)$ is also uniformly bounded. Thus, by using $(x+y)^{2} \geq \frac{1}{2} x^{2}-y^{2}$ and $A_{\infty}^{\alpha}=\sqrt{a_{\infty}^{\alpha}}=\sqrt{b_{\infty}^{\beta}}=B_{\infty}^{\beta}$ and the Cauchy-Schwarz inequality,

$$
\begin{aligned}
\left|\bar{A}^{\alpha}-\bar{B}\right|^{2} \geq & \frac{1}{2} A_{\infty}^{\alpha}\left|\prod_{i=1}^{M}\left(1+\mu_{i}\right)^{\alpha_{i}}-\prod_{j=1}^{N}\left(1+\zeta_{j}\right)^{\beta_{j}}\right|^{2} \\
& -|\Theta|^{2}(M+N)^{2}\left(\sum_{i=1}^{M}\left\|\delta_{i}\right\|^{2}+\sum_{j=1}^{N}\left\|\eta_{j}\right\|^{2}\right) .
\end{aligned}
$$

Hence, for any $\delta \in(0,1)$ holds

$$
\begin{aligned}
& \sum_{i=1}^{M}\left\|\delta_{i}\right\|^{2}+\sum_{j=1}^{N}\left\|\eta_{j}\right\|^{2}+\left|\bar{A}^{\alpha}-\bar{B} \beta\right|^{2} \\
& \geq \sum_{i=1}^{M}\left\|\delta_{i}\right\|^{2}+\sum_{j=1}^{N}\left\|\eta_{j}\right\|^{2} \\
& \quad+\delta\left(\frac{1}{2} A_{\infty}^{\alpha}\left|\prod_{i=1}^{M}\left(1+\mu_{i}\right)^{\alpha_{i}}-\prod_{j=1}^{N}\left(1+\zeta_{j}\right)^{\beta_{j}}\right|^{2}\right. \\
& \left.\quad-|\Theta|^{2}(M+N)^{2}\left(\sum_{i=1}^{M}\left\|\delta_{i}\right\|^{2}+\sum_{j=1}^{N}\left\|\eta_{j}\right\|^{2}\right)\right) \\
& \geq \frac{\delta}{2} A_{\infty}^{\alpha}\left|\prod_{i=1}^{M}\left(1+\mu_{i}\right)^{\alpha_{i}}-\prod_{j=1}^{N}\left(1+\zeta_{j}\right)^{\beta_{j}}\right|^{2}
\end{aligned}
$$

by choosing $\delta$ small enough such that $1 \geq \delta|\Theta|^{2}(M+N)^{2}$ since $\Theta$ is uniformly bounded above. This leads in combination with (62) to a lower bound of the left-hand side of (61)

$$
\text { LHS of }(61) \geq C_{1} \frac{\delta}{2} A_{\infty}^{\alpha}\left|\prod_{i=1}^{M}\left(1+\mu_{i}\right)^{\alpha_{i}}-\prod_{j=1}^{N}\left(1+\zeta_{j}\right)^{\beta_{j}}\right|^{2} .
$$

From (64) and (67), it is sufficient to prove

$$
\left|\prod_{i=1}^{M}\left(1+\mu_{i}\right)^{\alpha_{i}}-\prod_{j=1}^{N}\left(1+\zeta_{j}\right)^{\beta_{j}}\right|^{2} \geq C_{2}\left(\sum_{i=1}^{M} \mu_{i}^{2}+\sum_{j=1}^{N} \zeta_{j}^{2}\right) .
$$

In order to do so, we note that the conservation laws

$$
\beta_{j} \bar{a}_{i}+\alpha_{i} \bar{b}_{j}=\beta_{j} a_{i, \infty}+\alpha_{i} b_{j, \infty}
$$

rewritten in terms of the ansatz (63), i.e.

$$
\beta_{j} A_{i, \infty}^{2}\left(\mu_{i}^{2}+2 \mu_{i}\right)+\alpha_{i} B_{j, \infty}^{2}\left(\zeta_{j}^{2}+2 \zeta_{j}\right)=0 .
$$


imply $\mu_{i} \zeta_{j} \leq 0$ thanks to $\mu_{i}, \zeta_{j} \geq-1$ for all $i, j$. Without loss of generality, we assume $\mu_{i} \geq 0$ and $\zeta_{j} \leq 0$ for all $i, j$. Then, for any $1 \leq i_{0} \leq M$ and $1 \leq j_{0} \leq N$,

$$
\begin{aligned}
\left|\prod_{i=1}^{M}\left(1+\mu_{i}\right)^{\alpha_{i}}-\prod_{j=1}^{N}\left(1+\zeta_{j}\right)^{\beta_{j}}\right| & \geq \prod_{i=1}^{M}\left(1+\mu_{i}\right)^{\alpha_{i}}-\prod_{j=1}^{N}\left(1+\zeta_{j}\right)^{\beta_{j}} \\
& \geq\left(1+\mu_{i_{0}}\right)^{\alpha_{i_{0}}}-\left(1+\zeta_{j_{0}}\right)^{\beta_{j_{0}}} \\
& \geq\left(1+\mu_{i_{0}}\right)-\left(1+\zeta_{j_{0}}\right) \geq \mu_{i_{0}}-\zeta_{j_{0}} \geq 0 .
\end{aligned}
$$

Thus,

$$
\left|\prod_{i=1}^{M}\left(1+\mu_{i}\right)^{\alpha_{i}}-\prod_{j=1}^{N}\left(1+\zeta_{j}\right)^{\beta_{j}}\right|^{2} \geq\left(\mu_{i_{0}}-\zeta_{j_{0}}\right)^{2}=\mu_{i_{0}}^{2}-2 \mu_{i_{0}} \zeta_{j_{0}}+\zeta_{j_{0}}^{2} \geq \mu_{i_{0}}^{2}+\zeta_{j_{0}}^{2} .
$$

Since $1 \leq i_{0} \leq M$ and $1 \leq j_{0} \leq N$ are arbitrary, we finally obtain (68) with $C_{2}=1 / \max \{M ; N\}$.

Lemma 4.2. Let $a_{i}, b_{j}$ be functions defined in Lemma 4.1. Then, there exists a constant C such that

$$
\sum_{i=1}^{M}\left\|\delta_{i}\right\|^{2}+\sum_{j=1}^{N}\left\|\eta_{j}\right\|^{2}+\left\|A^{\alpha}-B^{\beta}\right\|^{2} \geq C\left|\bar{A}^{\alpha}-\bar{B}^{\beta}\right|^{2}
$$

Proof. Fix a constant $L>0$. Denote by

$$
S=\left\{x \in \Omega:\left|\delta_{i}(x)\right| \leq L,\left|\eta_{j}(x)\right| \leq L \text { for all } i=1, \ldots, M, j=1, \ldots, N\right\} \quad \text { and }
$$
$S^{\perp}=\Omega \backslash S$.

Recalling $\overline{A_{i}} \leq \sqrt{\overline{A_{i}^{2}}} \leq M_{0}$ and $\overline{B_{j}} \leq \sqrt{\overline{B_{j}^{2}}} \leq M_{0}$, we use Taylor expansion to estimate

$$
\begin{aligned}
\left\|A^{\alpha}-B^{\beta}\right\|^{2} & \geq \int_{S}\left|\prod_{i=1}^{M}\left(\bar{A}_{i}+\delta_{i}(x)\right)^{\alpha_{i}}-\prod_{j=1}^{N}\left(\bar{B}_{j}+\eta_{j}(x)\right)^{\beta_{j}}\right|^{2} \mathrm{~d} x \\
& \geq \frac{1}{2}\left|\bar{A}^{\alpha}-\bar{B}^{\beta}\right|^{2}|S|-\widetilde{R}\left(\bar{A}_{i}, \bar{B}_{j},\left|\delta_{i}\right|,\left|\eta_{j}\right|\right)\left(\sum_{i=1}^{M}\left\|\delta_{i}\right\|^{2}+\sum_{j=1}^{N}\left\|\eta_{j}\right\|^{2}\right)
\end{aligned}
$$

where $|\widetilde{R}| \leq C\left(M_{0}, L\right)$ due to the boundedness of $\delta_{i}$ and $\eta_{j}$ in $S$. In $S^{\perp}$, we have

$$
\sum_{i=1}^{M}\left\|\delta_{i}\right\|^{2}+\sum_{j=1}^{N}\left\|\eta_{j}\right\|^{2} \geq \int_{S^{\perp}}\left(\sum_{i=1}^{M}\left|\delta_{i}(x)\right|^{2}+\sum_{j=1}^{N}\left|\eta_{j}(x)\right|^{2}\right) \mathrm{d} x \geq L^{2}\left|S^{\perp}\right| .
$$

Next, there clearly exists a constant $\Lambda>0$ such that $\left|\bar{A}^{\alpha}-\bar{B}^{\beta}\right|^{2} \leq \Lambda$ since $\bar{A}_{i}, \bar{B}_{j} \leq$ $M_{0}$. Therefore, 


$$
\sum_{i=1}^{M}\left\|\delta_{i}\right\|^{2}+\sum_{j=1}^{N}\left\|\eta_{j}\right\|^{2} \geq L^{2}\left|S^{\perp}\right| \geq \frac{L^{2}}{\Lambda}\left|\bar{A}^{\alpha}-\bar{B}^{\beta}\right|^{2}\left|S^{\perp}\right| .
$$

Combining (69) and (70), we find for any $\theta_{1}, \theta_{2} \in(0,1)$

$$
\begin{aligned}
& \sum_{i=1}^{M}\left\|\delta_{i}\right\|^{2}+\sum_{j=1}^{N}\left\|\eta_{j}\right\|^{2}+\left\|A^{\alpha}-B^{\beta}\right\|^{2} \\
& \geq \theta_{1} \frac{L^{2}}{\Lambda}\left|\bar{A}^{\alpha}-\bar{B}^{\beta}\right|^{2}\left|S^{\perp}\right|+\left(1-\theta_{1}\right)\left(\sum_{i=1}^{M}\left\|\delta_{i}\right\|^{2}+\sum_{j=1}^{N}\left\|\eta_{j}\right\|^{2}\right) \\
& \quad+\theta_{2} \frac{1}{2}\left|\bar{A}^{\alpha}-\bar{B}^{\beta}\right|^{2}|S|-\theta_{2}|\widetilde{R}|\left(\sum_{i=1}^{M}\left\|\delta_{i}\right\|^{2}+\sum_{j=1}^{N}\left\|\eta_{j}\right\|^{2}\right) \\
& \geq \min \left\{\theta_{1} \frac{L^{2}}{\Lambda} ; \theta_{2} \frac{1}{2}\right\}\left|\bar{A}^{\alpha}-\bar{B}^{\beta}\right|^{2}\left(|S|+\left|S^{\perp}\right|\right) \\
& =\min \left\{\theta_{1} \frac{L^{2}}{\Lambda} ; \theta_{2} \frac{1}{2}\right\}\left|\bar{A}^{\alpha}-\bar{B}^{\beta}\right|^{2}
\end{aligned}
$$

by choosing $\theta_{1}, \theta_{2}$ small enough such that $1-\theta_{1}-\theta_{2}|\widetilde{R}| \geq 0$ and using $|S|+\left|S^{\perp}\right|=$ $|\Omega|=1$. The proof of Lemma 4.2 is hence complete.

\section{Proof Theorem 1.1: existence of global weak solution to (S)}

In this section, we give a proof Theorem 1.1 about the global existence of weak solutions to $(\mathrm{S})$ under the conditions $(\mathrm{G})-(\mathrm{M})-(\mathrm{P})$. Consider the approximating system

$$
\begin{gathered}
\partial_{t} u_{i, \varepsilon}-d_{i} \Delta\left(u_{i, \varepsilon}^{m_{i}}\right)=f_{i, \varepsilon}\left(u_{\varepsilon}\right):=\frac{f_{i}\left(u_{\varepsilon}\right)}{1+\varepsilon \sum_{i=1}^{S}\left|f_{i}\left(u_{\varepsilon}\right)\right|}, \\
\nabla\left(u_{i, \varepsilon}^{m_{i}}\right) \cdot \vec{n}=0, \quad u_{i, \varepsilon}(x, 0)=u_{i, 0, \varepsilon}(x)
\end{gathered}
$$

where $u_{\varepsilon}=\left(u_{1, \varepsilon}, \ldots, u_{S, \varepsilon}\right)$ and the sequence of approximating non-negative initial data $u_{i, 0, \varepsilon} \in L^{\infty}(\Omega)$ converges to $u_{i, 0}$ in $L^{2}(\Omega)$. By the construction of the approximative system, it directly follows that the nonlinearities $f_{i, \varepsilon}$ still satisfy the conditions (M) and (P). Moreover, for $\varepsilon>0$

$$
\left|f_{i, \varepsilon}\left(u_{\varepsilon}\right)\right| \leq \frac{\left|f_{i}\left(u_{\varepsilon}\right)\right|}{1+\varepsilon \sum_{i=1}^{S}\left|f_{i}\left(u_{\varepsilon}\right)\right|} \leq \frac{1}{\varepsilon} \quad \text { for all } u_{\varepsilon} \in \mathbb{R}^{S} .
$$

Hence, by a classical result for the porous medium equation with $L^{\infty}$ data, there exists a strong non-negative solution $u_{\varepsilon}=\left(u_{i, \varepsilon}\right)_{i=1 \ldots S}$ (see e.g. [43, Section 8]) in the sense that 


$$
\begin{aligned}
u_{i, \varepsilon}^{m_{i}} & \in L_{l o c}^{2}\left(0,+\infty ; H^{1}(\Omega)\right), \partial_{t} u_{i, \varepsilon}=d_{i} \Delta\left(u_{i, \varepsilon}^{m_{i}}\right)+f_{i, \varepsilon}\left(u_{\varepsilon}\right) \in L_{l o c}^{1}\left(0,+\infty ; L^{1}(\Omega)\right), \\
u_{i, \varepsilon} & \in C\left([0, T) ; L^{1}(\Omega)\right) \text { and } u_{i, \varepsilon}(0)=u_{i, 0, \varepsilon},
\end{aligned}
$$

and the equation for $u_{i, \varepsilon}$ holds a.e. in $Q_{T}$ for any $T>0$. Therefore, it follows immediately that

$$
\begin{aligned}
& -\int_{\Omega} u_{i, 0, \varepsilon} \psi(0) \mathrm{d} x-\int_{0}^{T} \int_{\Omega}\left(\partial_{t} \psi u_{i, \varepsilon}+u_{i, \varepsilon}^{m_{i}} \Delta \psi\right) \mathrm{d} x \mathrm{~d} t \\
& =\int_{0}^{T} \int_{\Omega} f_{i, \varepsilon}\left(u_{\varepsilon}\right) \psi \mathrm{d} x \mathrm{~d} t
\end{aligned}
$$

for any test function $\psi \in C^{2,1}(\bar{\Omega} \times[0, T])$ with $\psi(T)=0$ and $\nabla \psi \cdot \vec{n}=0$ on $\partial \Omega \times(0, T)$. As for the existence of weak solutions, it can be obtained by classical methods, for instance following the ideas in [1] and more precisely, derive a Lyapunov functional similar to the one on p. 39. One can also use similar arguments in [26, Proof of Lemma 2.3] with a few modifications to adapt to Neumann boundary conditions.

In order to pass to the limit as $\varepsilon \rightarrow 0$ in the weak formula (72), we use the following uniform a priori estimates, which are a consequence of a duality argument in the spirit of e.g. [36] and references therein.

Lemma 5.1. (Duality estimates and uniform a priori estimates for the approximating solutions, cf. [26]) Let $u_{\varepsilon}=\left(u_{1, \varepsilon}, \ldots, u_{S, \varepsilon}\right)$ be the non-negative solutions to the approximating system (71). Then,

$$
\left\|u_{i, \varepsilon}\right\|_{L^{m_{i}+1}\left(Q_{T}\right)} \leq C_{T} \text { for all } T>0 \text { and } i=1, \ldots, S,
$$

where the $\varepsilon$-independent constant $C_{T}$ depends only polynomially in T. Moreover, we have

$$
\left\|f_{i, \varepsilon}\left(u_{\varepsilon}\right)\right\|_{L^{1+\delta}\left(Q_{T}\right)} \leq C_{T}
$$

for some $\delta>0$, where the constant $C_{T}$ depends at most polynomially in $T>0$

Proof. The proof follows [26] with straightforward changes due to the considered Neumann (instead of Dirichlet) boundary conditions. By setting

$$
Z=\sum_{i=1}^{S} \lambda_{i} u_{i, \varepsilon} \quad \text { and } \quad W=\sum_{i=1}^{S} d_{i} \lambda_{i} u_{i, \varepsilon}^{m_{i}}
$$

and by summing up the equations of systems (S), the mass dissipation property (M) implies

$$
\partial_{t} Z-\Delta W \leq 0 \quad \text { and } \quad \nabla W \cdot \vec{n}=0
$$


Then, integration over $(0, t)$ and multiplication with $W(t)$ in $L^{2}(\Omega)$ (due to the regularity of the approximative solutions) lead after integration over $\Omega$ to

$$
\int_{\Omega}(Z(t)-Z(0)) W(t) \mathrm{d} x-\int_{\Omega} W(t) \Delta \int_{0}^{t} W(s) \mathrm{d} s \mathrm{~d} x \leq 0 .
$$

Next, we integrate by parts with homogeneous Neumann boundary conditions the second term on the left-hand side and calculate

$$
\begin{aligned}
-\int_{\Omega} W(t) \Delta \int_{0}^{t} W(s) \mathrm{d} s \mathrm{~d} x & =\int_{\Omega} \nabla W(t) \cdot \nabla \int_{0}^{t} W(s) \mathrm{d} s \mathrm{~d} x \\
& =\frac{1}{2} \frac{\mathrm{d}}{\mathrm{d} t} \int_{\Omega}\left|\nabla \int_{0}^{t} W(s) \mathrm{d} s\right|^{2} \mathrm{~d} x .
\end{aligned}
$$

Therefore, by integrating (74) with respect to $t$ on $(0, T)$, we obtain

$$
\int_{0}^{T} \int_{\Omega} Z(t) W(t) \mathrm{d} x \mathrm{~d} t+\frac{1}{2} \int_{\Omega}\left|\nabla \int_{0}^{T} W(s) \mathrm{d} s\right|^{2} \mathrm{~d} x \leq \int_{0}^{T} \int_{\Omega} Z(0) W(t) \mathrm{d} x \mathrm{~d} t .
$$

Moreover, we note that

$$
\begin{aligned}
\int_{0}^{T} \int_{\Omega} Z(t) W(t) \mathrm{d} x \mathrm{~d} t & =\int_{0}^{T} \int_{\Omega}\left(\sum_{i=1}^{S} \lambda_{i} u_{i, \varepsilon}\right)\left(\sum_{i=1}^{S} d_{i} \lambda_{i} u_{i, \varepsilon}^{m_{i}}\right) \mathrm{d} x \mathrm{~d} t \\
& \geq \sum_{i=1}^{S} d_{i} \lambda_{i}^{2}\left\|u_{i, \varepsilon}\right\|_{L^{m_{i}+1}\left(Q_{T}\right)}^{m_{i}+1}
\end{aligned}
$$

due to the non-negativity of functions $u_{i, \varepsilon}$ and the constant $\lambda_{i}$. To estimate the righthand side of (75) in terms of the $L^{2}$-norm of $Z(0)$, we first notice from $\partial_{t} Z-\Delta W \leq 0$ that

$$
Z(T)-\Delta \int_{0}^{T} W \mathrm{~d} t \leq Z(0)
$$

Define $\varphi(x)=\int_{0}^{T} W(x, t) \mathrm{d} t$, we have, thanks to $Z(T) \geq 0$,

$$
-\Delta \varphi \leq Z(0) \text { in } \Omega \text { and } \nabla \varphi \cdot \vec{n}=0 \text { on } \partial \Omega
$$

Multiplying this inequality by $\varphi \geq 0$ and using the Poincaré-Wirtinger inequality $\|\nabla \varphi\|^{2} \geq C_{P}\|\varphi-\bar{\varphi}\|^{2}$ yield

$$
\begin{aligned}
C_{P} & \|\varphi-\bar{\varphi}\|^{2} \leq\|\nabla \varphi\|^{2} \leq \int_{\Omega} Z(0) \varphi \mathrm{d} x \\
& =\int_{\Omega} Z(0)(\varphi-\bar{\varphi}) \mathrm{d} x+\bar{\varphi} \int_{\Omega} Z(0) \mathrm{d} x \\
& \leq \frac{C_{P}}{2}\|\varphi-\bar{\varphi}\|^{2}+\frac{1}{2 C_{P}}\|Z(0)\|^{2}+\bar{\varphi} \int_{\Omega} Z(0) \mathrm{d} x .
\end{aligned}
$$


where $\bar{\varphi}=\frac{1}{|\Omega|} \int_{\Omega} \varphi \mathrm{d} x$. Thus,

$$
\|\varphi-\bar{\varphi}\|^{2} \leq C\|Z(0)\|^{2}+\bar{\varphi}\|Z(0)\|_{L^{1}(\Omega)} .
$$

We can now estimate

$$
\begin{aligned}
\int_{0}^{T} \int_{\Omega} Z(0) W(t) \mathrm{d} x \mathrm{~d} t & =\int_{\Omega} \varphi Z(0) \mathrm{d} x=\int_{\Omega}(\varphi-\bar{\varphi}) Z(0) \mathrm{d} x+\bar{\varphi} \int_{\Omega} Z(0) \mathrm{d} x \\
& \leq 2\|\varphi-\bar{\varphi}\|^{2}+2\|Z(0)\|^{2}+\bar{\varphi} \int_{\Omega} Z(0) \mathrm{d} x \\
& \leq C\|Z(0)\|^{2}+C\|Z(0)\| \bar{\varphi}
\end{aligned}
$$

By inserting this into (75) and (76), we obtain

$$
\begin{aligned}
& \sum_{i=1}^{S} d_{i} \lambda_{i}^{2}\left\|u_{i, \varepsilon}\right\|_{L^{m_{i}+1}\left(Q_{T}\right)}^{m_{i}+1} \leq C\|Z(0)\|^{2}+C\|Z(0)\| \bar{\varphi} \\
& =C\|Z(0)\|^{2}+C\|Z(0)\| \sum_{i=1}^{S} d_{i} \lambda_{i}\left\|u_{i, \varepsilon}\right\|_{L^{m_{i}}\left(Q_{T}\right)}^{m_{i}} .
\end{aligned}
$$

An application of Young's inequality gives us the first a priori estimate (73) of Lemma 5.1.

Concerning the second uniform a priori estimate for the nonlinearities, we have

$$
\left|f_{i, \varepsilon}\left(u_{\varepsilon}\right)\right| \leq\left|f_{i}\left(u_{\varepsilon}\right)\right| \leq C\left(1+\left|u_{\varepsilon}\right|^{\nu}\right),
$$

where $C$ does not depend on $\varepsilon$. By the assumption $m_{i}>v-1$ and the estimate of $\left\|u_{i, \varepsilon}\right\|_{L^{m_{i}+1}\left(Q_{T}\right)}$, we obtain $\left\|f_{i, \varepsilon}\left(u_{\varepsilon}\right)\right\|_{L^{1+\delta}\left(Q_{T}\right)} \leq C_{T}$.

The following compactness lemma allows to extract a converging subsequence from the approximating system.

Lemma 5.2. [3] Let $m>(d-2)_{+} / d$ with $(d-2)_{+}=\max \{0, d-2\}$. The mapping $L^{1}(\Omega) \times L^{1}\left(Q_{T}\right) \ni\left(u_{0}, f\right) \mapsto u \in L^{1}\left(Q_{T}\right)$ where $u \in C\left([0, T] ; L^{1}(\Omega)\right)$ is the weak solution to

$$
\partial_{t} u-\delta \Delta\left(u^{m}\right)=f, \quad \nabla\left(u^{m}\right) \cdot \vec{n}=0, \quad u(0)=u_{0},
$$

with $\delta>0$, is compact.

Proof of Theorem 1.1. Thanks to the uniform bounds of the nonlinearities in Lemma 5.1 and the compactness Lemma 5.2, there exists a subsequence (not relabelled) $\left\{u_{i, \varepsilon}\right\}_{\varepsilon}$ which converges in $L^{1}\left(Q_{T}\right)$ to limit functions $u_{i} \in L^{1}\left(Q_{T}\right)$. From the $L^{m_{i}+1}$-bound in Lemma 5.1, it holds in fact that $u_{i, \varepsilon}$ (up to another subsequence) converges strongly to $u_{i}$ in $L^{m_{i}}\left(Q_{T}\right)$. For the nonlinearities, we first notice from Lemma 5.1 that the sequence $\left\{f_{i, \varepsilon}\left(u_{\varepsilon}\right)\right\}$ is uniformly integrable. Moreover, for another subsequence $u_{i, \varepsilon} \rightarrow u_{i}$ a.e. in $Q_{T}$, it follows that

$$
f_{i, \varepsilon}\left(u_{\varepsilon}\right) \rightarrow f_{i}\left(u_{i}\right) \quad \text { a.e. in } \quad Q_{T} .
$$


Therefore, we can apply Vitali's Lemma, see e.g. [41, Chapter 16], to obtain $f_{i, \varepsilon}\left(u_{\varepsilon}\right) \rightarrow$ $f_{i}\left(u_{i}\right)$ strongly in $L^{1}\left(Q_{T}\right)$. All this allows to pass to the limit in the weak formulation (72) for any test function $\psi \in C^{2,1}(\bar{\Omega} \times[0, T])$ with $\psi(T)=0$ and $\nabla \psi \cdot \vec{n}=0$ on $\partial \Omega \times(0, T)$. Hence, we get

$$
-\int_{\Omega} \psi(0) u_{i, 0} \mathrm{~d} x-\int_{Q_{T}}\left(\partial_{t} \psi u_{i}+u_{i}^{m_{i}} \Delta \psi\right) \mathrm{d} x \mathrm{~d} t=\int_{Q_{T}} f_{i}(u) \psi \mathrm{d} x \mathrm{~d} t .
$$

The additional regularity $u_{i}^{m_{i}} \in L^{1}\left(0, T ; W^{1,1}(\Omega)\right)$ follows immediately from [29, Lemma 4.7], ${ }^{1}$ where

$\int_{0}^{T} \int_{\Omega}\left|\nabla u_{i}^{m_{i}}\right|^{\beta} \mathrm{d} x \mathrm{~d} t \leq C\left(T,\left\|u_{i, 0}\right\|_{1},\left\|f_{i}(u)\right\|_{L^{1}\left(Q_{T}\right)}\right) \quad$ for all $1 \leq \beta<1+\frac{1}{1+m_{i} d}$.

From the above estimate and $f_{i}(u) \in L^{1}\left(Q_{T}\right)$, we also have $\partial_{t} u_{i} \in L^{1}\left(0, T ;\left(W^{1,1}(\Omega)\right)^{*}\right)$ which implies in particular $u_{i} \in C\left([0, T] ; L^{1}(\Omega)\right)$. This completes the proof of existence of global weak solutions.

\section{Acknowledgements}

Open access funding provided by University of Graz. We would like to thank the referee for the comments and suggestions which help to significantly improve the paper. The second author was supported by the DFG Project CH 955/3-1. This work is partially supported by International Research Training Group IGDK 1754 and NAWI Graz.

Open Access. This article is distributed under the terms of the Creative Commons Attribution 4.0 International License (http://creativecommons.org/licenses/by/4.0/), which permits unrestricted use, distribution, and reproduction in any medium, provided you give appropriate credit to the original author(s) and the source, provide a link to the Creative Commons license, and indicate if changes were made.

Publisher's Note Springer Nature remains neutral with regard to jurisdictional claims in published maps and institutional affiliations.

\section{REFERENCES}

[1] D. G. Aronson, "The porous medium equation, Nonlinear diffusion problems (Montecatini Terme, 1985)", Springer, Lecture Notes in Math. 1224, (1985) 1-46.

[2] A. Barabanova, "On the global existence of solutions of a reaction-diffusion system with exponential nonlinearity”, Proc. Am. Math. Soc. 122, (1994) 827-831.

[3] P. Baras, "Compacité de l'opérateur $f \mapsto u$ solution d'une équation non linéaire $\frac{d u}{d t}+A u \ni f$." C. R. Acad. Sci., Sér. A 286 (1978) 1113-1116.

[4] S. Benachour, B. Rebiai, "Global classical solutions for reaction-diffusion systems with nonlinearities of exponential growth", J. Evol. Equ. 10 (2010) 511-527.

[5] N. Boudiba and M. Pierre, "Global existence for Coupled Reaction-Diffusion Systems". J. Math. Anal. Appl. 250 (2000) 1-12.

\footnotetext{
${ }^{1}$ The results presented in [29] are for homogeneous Dirichlet boundary conditions. However, similar results for homogeneous Neumann boundary conditions can be obtained with slight modifications.
} 
[6] C. Caputo and A. Vasseur, "Global Regularity of Solutions to Systems of Reaction-Diffusion with Sub-Quadratic Growth in Any Dimension". Comm. Partial Differential Equations, 34.10 (2009) 1228-1250.

[7] M.C. Caputo, T. Goudon, A. Vasseur, "Solutions of the 4-species quadratic reaction-diffusion system are bounded and $C^{\infty}$-smooth, in any space dimension”, Anal. PDE Vol. 12, Nr 7, (2019), 1773-1804.

[8] J.A. Cañizo, L. Desvillettes, K. Fellner, "Improved duality estimates and applications to reactiondiffusion equations." Comm. Partial Differential Equations 39.6 (2014), 1185-1204.

[9] E. DiBenedetto, A. Friedman, "Hölder estimates for nonlinear degenerate parabolic systems." J. Reine Angew. Math., 357 (1985) 1-22.

[10] L. Desvillettes, K. Fellner, "Exponential decay toward equilibrium via entropy methods for reactiondiffusion equations.” J. Math. Anal. Appl. 319.1 (2006) 157-176.

[11] L. Desvillettes, K. Fellner, "Entropy methods for reaction-diffusion systems." Discrete Contin. Dyn. Syst. (suppl). Proceedings of the 6th AIMS International Conference (2007) 304-312.

[12] L. Desvillettes, K. Fellner, "Entropy methods for reaction-diffusion equations: slowly growing a-priori bounds." Rev. Mat. Iberoamericana 24.2 (2008) 407-431.

[13] L. Desvillettes, K. Fellner, B.Q. Tang, "Trend to equilibrium for reaction-diffusion systems arising from complex balanced chemical reaction networks.” SIAM J. Math. Anal. 49 (2017) 2666-2709.

[14] L. Desvillettes, K. Fellner, M. Pierre, J. Vovelle, "About Global Existence for Quadratic Systems of Reaction-Diffusion”, Adv. Nonlinear Stud. 7 (2007) 491-511.

[15] K. Fellner, E. Latos, T. Suzuki, "Global classical solutions for mass-conserving, (super)-quadratic reaction-diffusion systems in three and higher space dimensions", Discrete and Continuous Dynamical Systems - Series B. 21.10 (2016) 3441-3462.

[16] K. Fellner, J. Morgan, B.Q. Tang, "Global classical solutions to quadratic systems with mass control in arbitrary dimensions". to appear in Ann. Inst. H. Poincaré Anal. Non Linéaire.

[17] K. Fellner, E. Latos, B.Q. Tang, "Well-posedness and exponential equilibration of a volume-surface reaction-diffusion system with nonlinear boundary coupling." Ann. Inst. H. Poincaré Anal. Non Linéaire. 35.3 (2018) 643-673.

[18] K. Fellner, W. Prager, B.Q. Tang, "The entropy method for reaction-diffusion systems without detailed balance: first order chemical reaction networks.”, Kinet. Relat. Models. 10 (4) (2017) 1055-1087.

[19] K. Fellner, B.Q. Tang, "Explicit exponential convergence to equilibrium for nonlinear reactiondiffusion systems with detailed balance condition." Nonlinear Anal. 159 (2017) 145-180.

[20] K. Fellner, B.Q. Tang, "Convergence to equilibrium for renormalised solutions to nonlinear chemical reaction-diffusion systems", Zeitschrift für angewandte Mathematik und Physik. 69.3 (2018) (30 pages).

[21] W.B. Fitzgibbon, S.L. Hollis and J.J. Morgan, Stability and Lyapunov Functions for ReactionDiffusion Systems, SIAM J. Math. Ana., 28 No 3 (1997), 595-610.

[22] M. A. Herrero, A. A. Lacey and J. L. Velázquez, "Global Existence for Reaction-Diffusion Systems Modelling Ignition", Arch. Rational Mech. Anal. 142 (1998) 219-251.

[23] S.L. Hollis, R.H. Martin, M. Pierre, "Global existence and boundedness in reaction-diffusion systems.” SIAM J. Math. Anal. 18(3) (1987) 744-761.

[24] J.I. Kanel, M. Kirane, "Global solutions of reaction-diffusion systems with a balance law and nonlinearities of exponential growth”, J. Differential Equations 165 (2000) 24-41.

[25] E.-H. Laamri, "Global existence of classical solutions for a class of reaction-diffusion systems", Acta Appl. Math. 115(2) (2011) 153-165.

[26] E.H. Laamri, M. Pierre, "Global existence for reaction-diffusion systems with nonlinear diffusion and control of mass.” Ann. Inst. H. Poincaré Anal. Non Linéaire. 34.3 (2017) 571-591.

[27] O.A. Ladyženskaja, V.A. Solonnikov, N.N. Ural'ceva, "Linear and quasilinear equations of parabolic type". American Mathematical Society, Providence. 1968.

[28] A.W. Leung, "Nonlinear Systems of Partial Differential Equations: Applications to Life and Physical Sciences.” World Scientific, Singapore. 2009.

[29] T. Lukkari, “The porous medium equation with measure data.” J. Evol. Equ. 10 (2010) 711-729. 
[30] R.H. Martin and M. Pierre, "Nonlinear reaction-diffusion systems in Nonlinear Equations in the Applied Sciences", W.F. Ames and C. Rogers ed., Math. Sci. Eng. 185, Acad. Press, New York 1991.

[31] K. Masuda, "On the global existence and asymptotic behavior of reaction-diffusion equations", Hokkaido Math. J. 12 (1983) 360-370.

[32] A. Mielke, "Uniform exponential decay for reaction-diffusion systems with complex-balanced mass-action kinetics." Patterns of dynamics, 149-171, Springer Proceedings in Mathematics \& Statistics, 205, Springer, Cham, 2017.

[33] A. Mielke, J. Haskovec, P. A. Markowich, "On uniform decay of the entropy for reaction-diffusion systems”, J. Dynam. Differential Equations, 27 (2015) 897-928.

[34] A. Mielke, M. Mittnenzweig, "Convergence to equilibrium in energy-reaction-diffusion systems using vector-valued functional inequalities.” J. Nonlinear Sci. 28(2) (2018), 765-806.

[35] J. Morgan, "Global existence for semilinear parabolic systems.” SIAM J. Math. Anal. 20(5) (1989) 1128-1144.

[36] M. Pierre, "Global existence in reaction-diffusion systems with control of mass: a survey." Milan J. Math. 78.2 (2010) 417-455.

[37] M. Pierre, "Weak solutions and supersolutions in $L^{1}$ for reaction-diffusion systems", J. Evol. Equ. 3 (2003) 153-168.

[38] M. Pierre, G. Rolland, "Global existence for a class of quadratic reaction-diffusion systems with nonlinear diffusions and $L^{1}$ initial data.” Nonlinear Anal. 138 (2016) 369-387.

[39] M. Pierre and D. Schmitt, "Blow up in reaction-diffusion systems with dissipation of mass", SIAM J. Math. Anal. 28, (1997) 259-269.

[40] M. Pierre, T. Suzuki and R. Zou, "Asymptotic behavior of solutions to chemical reaction-diffusion systems.” J. Math. Anal. Appl., 450.1 (2017) 152-168.

[41] R.L. Schilling, "Measures, Integrals and Martingales." Cambridge University Press, Cambridge. 2005.

[42] J. Smoller, "Shock Waves and Reaction-Diffusion Equations." Springer, Berlin. 1994.

[43] J. Vázquez, "The porous medium equation: Mathematical Theory”, Oxford Science Publications, Oxford. 2007.

\author{
Klemens Fellner and Bao Quoc Tang \\ Institute of Mathematics and Scientific Computing \\ University of Graz. \\ Heinrichstrasse 36 \\ 8010 Graz \\ Austria \\ E-mail: klemens.fellner@uni-graz.at \\ Bao Quoc Tang \\ E-mail: quoc.tang@uni-graz.at \\ Evangelos Latos \\ University of Mannheim \\ 68131 Mannheim \\ Germany \\ E-mail: evangelos.latos@math.uni-mannheim.de
}

